\title{
Auditory deviance detection in the human insula: An intracranial EEG study
}

doi: https://doi.org//0.1 101/487306

\section{Author Names and affiliations:}

Alejandro O. Blenkmann ${ }^{a^{*}}$, Santiago Collavini ${ }^{b}$, James Lubell a , Anaïs Llorens ${ }^{\text {a,c }}$, Ingrid Funderud a, Jugoslav Ivanovic $^{\text {c }}$, Pål G. Larsson ${ }^{\text {c }}$, Torstein R. Meling ${ }^{c}$, Tristan Bekinschtein ${ }^{\mathrm{d}}$, Silvia Kochen ${ }^{\mathrm{b}}$, Tor Endestad ${ }^{\mathrm{a}, \mathrm{f}}$, Robert T. Knight $^{\mathrm{e}}$, Anne-Kristin Solbakk ${ }^{\mathrm{a}, \mathrm{c}, \mathrm{f}}$
a. Department of Psychology, University of Oslo, Norway
b. Studies in Neurosciences and Complex Systems, National Scientific and Technical Research Council - El Cruce Hospital - Arturo Jauretche National University, Argentina
c. Department of Neurosurgery, Oslo University Hospital - Rikshospitalet, Norway
d. Psychology Department, University of Cambridge, UK
e. Helen Wills Neuroscience Institute and Department of Psychology, University of California at Berkeley, USA
f. Department of Neuropsychology, Helgeland Hospital, Mosjøen, Norway

\section{Author e-mail addresses:}

Alejandro O. Blenkmann - ablenkmann@gmail.com

Santiago Collavini - santiagocollavini@gmail.com

James Lubell - sour.rye@gmail.com

Anaïs Llorens - anaisllorens@hotmail.com

Ingrid Funderud - ingridfunderud@hotmail.com

Jugoslav Ivanovic - jugiva@ous-hf.no

Pål G. Larsson - pall@ous-hf.no

Torstein R. Meling - torsteinrmeling@gmail.com

Tristan Bekinschtein - trisbek@gmail.com

Silvia Kochen - skochen@gmail.com

Tor Endestad - tor.endestad@psykologi.uio.no

Robert T. Knight - rtknight@berkeley.edu

Anne-Kristin Solbakk - a.k.solbakk@psykologi.uio.no

Corresponding author: Alejandro Blenkmann, Dept. of Psychology, University of Oslo, P.O. Box 1094 Blindern, 0317 Oslo, Norway 


\section{ABSTRACT}

The human insula is known to be involved in auditory processing but knowledge about its precise functional role and the underlying electrophysiology is limited. To assess its role in automatic auditory deviance detection we analyzed the EEG high frequency activity (HFA; 75-145 Hz) and ERPs from 90 intracranial insular channels across 16 patients undergoing pre-surgical intracranial monitoring for epilepsy treatment. Subjects passively listened to a stream of standard and deviant tones differing in four physical dimensions: intensity, frequency, location or time. HFA responses to auditory stimuli were found in the short and long gyri, and the anterior, superior, and inferior segments of the circular sulcus of the insular cortex. Only a subset of channels in the inferior segment of the circular sulcus of the insula showed HFA deviance detection responses, i.e. a greater and longer latency response to specific deviants relative to standards. Auditory deviancy processing was also later in the insula when compared with the superior temporal cortex. ERP results were more widespread and supported the HFA insular findings. These results provide evidence that the human insula is engaged during auditory deviance detection.

\section{INTRODUCTION}

Information on the role of the human insula in auditory processing is limited. Evidence from intracranial EEG (iEEG) indicates that the insula plays a role in auditory processing, and that insular electrical stimulation elicits simple auditory illusions and hallucinations (Afif, Minotti, Kahane, \& Hoffmann, 2010; Zhang et al., 2019). Other studies reported auditory agnosia after bilateral insular lesion (Bamiou, Musiek, \& Luxon, 2003), and deficits in the temporal resolution and sequencing of sounds after unilateral insular stroke (Bamiou et al., 2006). Additionally, insular neurons fire in response to auditory stimuli in non-human primates (Bieser, 1998; Remedios, Logothetis, \& Kayser, 2009), which share similar auditory cortex architectonics with humans (Fullerton \& Pandya, 2007; Galaburda \& Sanides, 1980).

Responses to unexpected sounds in regular auditory streams have been extensively studied with the mismatch negativity (MMN), an event-related potential (ERP) that peaks around 150-250 ms after the onset of an infrequent acoustic stimulus (e.g., Näätänen, Paavilainen, Rinne, \& Alho, 2007). MMN is considered a prediction error signal reflecting automatic change detection (Bubic, 2010; Clark, 2013). Multiple iEEG studies have shown that the superior temporal cortex is involved in deviance detection (Edwards, Soltani, Deouell, Berger, \& Knight, 2005; El Karoui et al., 2015; Halgren et al., 1995; Phillips, Blenkmann, Hughes, Kochen, Bekinschtein, Cam-CAN, et al., 2016), as well as the lateral prefrontal cortex and the nucleus accumbens (Durschmid et al., 2016a, 2016b). Previous fMRI (Nazimek, Hunter, Hoskin, Wilkinson, \& Woodruff, 2013; Sabri, Kareken, Dzemidzic, Lowe, \& Melara, 2004; Schall, Johnston, Todd, Ward, \& Michie, 2003), PET (Müller, Jüptner, Jentzen, \& Müller, 2002), and MEG studies (Lappe, Steinsträter, \& Pantev, 2013) have indicated that the insula plays a role in automatic deviance detection. However, a high spatial and temporal resolution electrophysiological analysis of the role of the insula in auditory processing is lacking. We addressed this by analyzing High Frequency Activity (HFA; 75-145 Hz) and ERPs in iEEG recordings. HFA is a reliable electrophysiological correlate of underlying averaged spiking activity generated by the thousands of neurons that are in the immediate vicinity of the recording electrodes (Lachaux, Axmacher, Mormann, Halgren, \& Crone, 2012; Ray \& Maunsell, 2011; Rich 
\& Wallis, 2017; Watson, Ding, \& Buzsáki, 2018), whereas ERPs reflect a mixture of local potentials and volume conducted potentials from distant sites (Lachaux et al., 2012). Nevertheless, the MMN is the most common ERP used to describe deviance detection. In this study of auditory deviance detection in the human insula we focus on the analysis of the HFA responses, describe the ERPs in the HFA sensitive areas, and compare the insular responses with the superior temporal cortex.

\section{METHODS}

We report how we determined our sample size, all data exclusions, all inclusion/exclusion criteria, whether inclusion/exclusion criteria were established prior to data analysis, all manipulations, and all measures in the study.

\subsection{Ethics statement}

This study was approved by the Research Ethics Committee of El Cruce Hospital, Argentina, and the Regional Committees for Medical and Health Research Ethics, Region North Norway. Patients gave informed written consent prior to participation. No part of the study procedures or analyses was pre-registered in a time-stamped, institutional registry prior to the research being conducted.

\subsection{Participants}

We recorded data from 22 normal hearing adults with drug-resistant epilepsy who were potential candidates for resective surgery. Insula coverage was the inclusion criteria for this study, resulting in 16 datasets with insula coverage for detailed analysis ( 6 female, mean age $=31$, range 19-50 years). Patients underwent invasive stereoelectroencephalography (SEEG) recordings as part of their pre-surgical evaluation. Intracranial depth electrodes were temporarily implanted to localize the epileptogenic zone and eloquent cortex. Data were collected at El Cruce Hospital or Oslo University Hospital. Patients were implanted with depth electrodes of 8-18 contacts with 1.5-5 mm inter-electrode distance (AdTech, USA, and DIXI Medical, France).

\subsection{Task}

A multi-dimensional auditory oddball paradigm was used (Näätänen, Pakarinen, Rinne, \& Takegata, 2004; Phillips et al., 2016). Standard tones were defined across four dimensions: frequency, intensity, location and time. Standards were interleaved with deviant tones that deviated in one of the four dimensions, while holding other stimulus dimensions constant. Deviants of type Freq-High and Freq-Low were higher or lower in frequency, Int-Up and Int-Down were louder or softer in intensity, Loc-Right and Loc-Left had the location of the sound source on the right or left, Time-Dur were shorter in duration, and Time-Gap had a silent gap in the middle of the tone. Table 1 depicts the most relevant stimulus features for all conditions. Tones had a duration of $75 \mathrm{~ms}$ (except TimeDur deviants), and were presented every $500 \mathrm{~ms}$, in blocks of 5 minutes consisting of 300 standard and 300 deviant tones. At the beginning of each block, fifteen standards were played. Deviants were presented in pseudo-random order such that a deviant type never appeared twice in a row (Figure 1A). Participants were asked not to pay attention to the sounds while reading a book or magazine. They completed 3 to 10 blocks, providing at least 1800 trials. The tones were presented through headphones using Psychtoolbox-3 (Kleiner, Brainard, 2007) for Matlab (The MathWorks Inc., USA). 


\subsection{Data acquisition}

Pre-implantation structural MRI and post-implantation CT scans were acquired for each participant. SEEG data were recorded using an Elite (Blackrock NeuroMed LLC, USA), a NicoletOne (Nicolet, Natus Neurology Inc., USA), or an ATLAS (Neuralynx, USA) system with sampling frequencies of 2000, 512, and $16000 \mathrm{~Hz}$, respectively.

\subsection{Electrode localization}

Post-implantation CT images were coregistered to pre-implantation MRI images using SPM12 (Studholme, Hill, \& Hawkes, 1999). MRI images were processed using the FreeSurfer standard pipeline (Dale, Fischl, \& Sereno, 1999), and individual cortical parcellation images were obtained using the Destrieux atlas (Destrieux, Fischl, Dale, \& Halgren, 2010). Images were spatially normalized to the MNI-152 template using SPM12 (Ashburner \& Friston, 2005) and electrode coordinates were obtained using the iElectrodes toolbox (Blenkmann et al., 2017). Anatomical labels were automatically assigned to each contact based on the Destrieux atlas using the aforementioned toolboxes, and confirmed by a neurologist (RTK).

\subsection{Signal pre-processing}

Monopolar iEEG recordings were manually inspected and channels or epochs showing epileptiform activity or abnormal signal were removed. Signals from electrodes located in lesional tissue or tissue that was later resected were also excluded. Bipolar channels were computed as the difference between signals recorded from pairs of neighboring electrodes in the same electrode array.

Subsequently, we refer to the bipolar channels as "channels". Data were low-pass filtered at $180 \mathrm{~Hz}$ and line noise was removed using bandstop filters at 50, 100, and $150 \mathrm{~Hz}$. Data were then segmented into $2000 \mathrm{~ms}$ epochs ( $-750 \mathrm{~ms}$ before and $1250 \mathrm{~ms}$ after tone onset) and demeaned. We manually inspected and rejected epochs after re-referencing. In order to eliminate any residual artifact, we rejected trials with an amplitude larger than 5 standard deviations from the mean for more than 25 consecutive ms, or with a power spectral density above 5 standard deviations from the mean for more than 6 consecutive Hz. An average of $35 \%$ of the trials were rejected, resulting in an average of 1592 trials analyzed per patient (range 728 to 3723 trials). Data were resampled to 1000 Hz. Pre-processing and statistical analysis were performed in Matlab using the Fieldtrip toolbox (Oostenveld, Fries, Maris, \& Schoffelen, 2011) and custom code.

\subsection{High frequency activity (HFA) extraction}

Preprocessed data were bandpass filtered into eight bands of $10 \mathrm{~Hz}$ bandwidth ranging from 75 to $145 \mathrm{~Hz}$.

The Hilbert transform was applied to each filtered signal to create the complex-valued analytic time series, and the modulus of these signals computed to retain the analytic amplitude time series. These envelopes are not affected by the polarity of the signals that constitute the bipolar channels. To compensate for the $1 / \mathrm{f}$ spectral trend, the mean amplitude of the baseline period ( -100 to $0 \mathrm{~ms})$ of each trial and frequency band was removed from the entire trial. The mean of the eight frequency bands was computed such that a single time series was built per trial. Finally, for each channel, all trial time series were divided by the standard deviation pulled from all trials in the baseline period, thereby computing the HFA time series of each channel as a normalized measure relative to the baseline activity. 


\subsection{Region of interest channel selection}

We studied channels in the insular cortex categorized into the following sub-areas based on Duvernoy's anatomical nomenclature (Destrieux et al., 2010; Duvernoy, 1999; Figure 1A-C: i) short insular gyri (SG), ii) long insular gyrus and central insular sulcus (LG), iii) anterior segment of the circular sulcus (aCS), iv) superior segment of the circular sulcus (sCS), and v) inferior segment of the circular sulcus (iCS). Ninety insular channels were analyzed across 16 patients (median 4.5, range 1-21 channels per patient). The distribution of channels across sub-areas is shown in Figure 1E and across patients in Table S1.

To compare responses from the insular cortex with the temporal cortex, we studied 93 channels from the superior temporal sulcus (STS) and 19 channels from the superior temporal gyrus (STG) across 14 of the 16 patients (See details in section S.5.1 and Table S1).

\subsection{Tone-responsive channels}

Channels were considered Tone-responsive if the median HFA response to tones, irrespective of type, was statistically larger in the post-stimulus period (0 to $300 \mathrm{~ms}$ after stimulus onset) relative to the baseline period (-100 to $0 \mathrm{~ms}$ ) (Durschmid et al., 2016a). We tested the differences in five 100 ms overlapping windows in the post-stimulus period, with $50 \%$ overlap, using a permutation-based approach. For each channel, we computed the difference between the HFA medians in the poststimulus windows and the HFA medians in the baseline period. We then created an empirical distribution by circular-shifting of the HFA trial time series (between -100 and $500 \mathrm{~ms}$ ) by a random number of samples. This allows any time-locked neural activity to be teased apart. Differences between the medians in the post-stimulus and baseline periods were measured for each surrogate set of trials. The procedure was repeated 1000 times and a null distribution of differences was built. Channels exceeding the 97.5th percentile of the channel-specific surrogate distribution in any of the five windows were considered showing a significant time-locked HFA modulation and were marked as Tone-responsive channels.

\subsection{Condition-specific channels}

To determine whether the Tone-responsive channels were sensitive to intensity, frequency, location or time deviances, we performed for each channel four one-way ANOVA cluster-based permutation tests with 3 levels: the standard tones and the two deviant tones of each dimension (alpha $=0.05$, 1000 permutations). For example, for the intensity dimension we tested in each channel the responses to Standards, Int-Up, and Int-Down tones. Post-hoc one-tailed t-tests were performed to determine if the HFA responses to deviants were higher in amplitude than those to standards (cluster-based permutation test, alpha $=0.025,1000$ permutations).

\subsection{HFA responses latency analysis}

The latency of the HFA responses were analyzed using the fractional area latency method (Luck, 2005). Briefly, the cumulative area under the HFA responses within a $-50 \mathrm{~ms}$ to $400 \mathrm{~ms}$ temporal window were computed and normalized between 0 and 100\%. Latency differences between conditions or brain areas were tested at the $50 \%$ fractional area (Wilcoxon signed rank test or Wilcoxon rank-sum test respectively). This latency is the time point where the area under the curve is equal on both sides of the response. 


\subsection{ERP analysis}

Low frequency signals $(0.01$ to $30 \mathrm{~Hz})$ were extracted, ERPs computed and analyzed to obtain Tone-responsive and Condition-specific channels in a similar way as the HFA analysis. Using PCA, we identified groups of responses with consistent temporal patterns across channels and subjects. See methodological details in section S.3.

\section{RESULTS}

When queried, all participants reported that they were able to focus on the reading material and did not attend to the tones. They did not notice any pattern in the stream of tones.

\subsection{Auditory effects}

In the HFA analysis, 29 insular channels responded to tones (all trials) in 11 out of the 16 patients (mean 2.6, range 1-5 channels per patient, $\mathrm{p}<0.025$, Figure 1B-C). Figure 2A shows the mean HFA response of one illustrative channel, along with the single trial HFA, and Figure $3 \mathrm{~A}$ the mean across all Tone-responsive channels. The median peak response latency was $120 \mathrm{~ms}, 95 \%$ CI [100, 141] $\mathrm{ms}$. Channels showing a significant response were non-uniformly distributed in all subdivisions of the insula; the predominant number of channels were in the iCS (Figure 1E; Fisher's exact test, $p=$ $0.02)$.

The ERP analysis showed auditory effects in 76 insular channels across 15 patients, including 28 out of the 29 HFA Tone-responsive channels ( $<<0.05$, Figure S1). The strongest ERP responses were located in the iCS (see details in section S.4.2, and the ERP responses to tones in Figure S2). Analysis in the superior temporal cortex showed 30 HFA Tone-responsive channels across 9 patients in the STS, and 12 channels across 6 subjects in the STG (Figure 3A). A larger proportion of the recorded channels was Tone-responsive in STG (63\%) in relationship to insula (32\%) and STS $(33 \%$, Fisher's exact test, $p=0.035)$. A 50\% area latency analysis indicated that responses to tones in the insula occurred after STS and STG $(164,133$, and $140 \mathrm{~ms}$ respectively, statistical details in section S.5.2 and Table S2). Figure 3B shows the mean fractional area latency for the responses to all tones for the three cortical areas. Figure S4A-B shows the equivalent for standard and deviant tone responses.

\subsection{Deviance detection effects}

To obtain a global description of the role of the insula in deviance detection we tested the HFA response to standards versus deviants (across dimensions) in all Tone-responsive channels. Deviants showed a larger response in the $134-196 \mathrm{~ms}$ interval after tone onset $(\mathrm{p}=0.014$, Figure 3C). Similarly, statistical differences were observed in the iCS ERP responses. An intracranial ERP equivalent to the scalp MMN potential depicted peaks at 142 and $217 \mathrm{~ms}$ (see details in section S.4.3, and the ERP responses to standard and deviant tones in Figure S3).

Eight channels across five patients showed HFA condition-specific effects to one or more of the deviant dimensions $(\mathrm{p}<0.05)$. All Condition-specific channels were located in the iCS (Figures 1BE), which represent $15 \%$ of the recorded, and $32 \%$ of the Tone-responsive channels in the area. Figure 2B shows the HFA responses to all stimulus types in one illustrative channel. Across the eight channels, seven had significantly higher responses to at least one deviant condition compared to standards (post-hoc permutation test). For illustration, we computed the mean HFA time course 
(averaged across significant channels) of each deviant condition and its corresponding standard tone response (Figure 4). To summarize the condition-specific effects from individual channel statistics, we computed the average of Cohen's d effect size across channels (Figure 4, top images). Effects sizes above medium $(0.5)$ or large $(0.8)$ were observed for all conditions in the HFA analysis (Cohen, 1992). Loc-Left $(\mathrm{p}<0.001)$ and Loc-Right $(\mathrm{p}<0.001)$ deviant responses showed similar time courses independent of tone laterality. Responses for Freq-High $(\mathrm{p}<0.001)$ and Freq-Low $(\mathrm{p}$ $<0.05)$ deviants were also similar. The average effect sizes for Int-Up $(\mathrm{p}<0.001)$ were larger than for the Int-Down $(\mathrm{p}<0.05)$, but Int-Down elicited a larger response than standards, suggesting that the HFA response was not solely driven by tone intensity. Time-Gap $(\mathrm{p}<0.001)$ and Time-Dur $(\mathrm{p}<$ 0.05 ) deviants yielded different HFA time courses. Additionally, one channel showed a higher HFA response to standards when compared to Int-Down deviants. Condition-specific responses were concurrent in some channels. Three of the channels were sensitive to all stimuli dimensions, one channel to three dimensions, one channel to two dimensions, and two channels to only one dimension (Figure 1B-C).

ERP condition-specific effects were present in 48 channels across 12 patients, including all HFA Condition-specific channels ( $p<0.05$, see details in section S.4.4 and Figure S1 for the spatial distribution of these channels). Figure 5 shows the ERP responses to each type of deviant and the corresponding standard tones for the two groups of channels in the iCS (see details in section S.4.3). Condition-specific differences in Group 1 were observed to be stronger after $180 \mathrm{~ms}$, whereas for channels in Group 2 differences were more predominantly observed between 100 and $250 \mathrm{~ms}$. Cohen's d effect size $(>-0.7$ and $<0.7)$ for condition-specific effects were on average smaller for the ERP analysis.

STS and STG cortices elicited larger HFA responses to deviant tones when compared to standard tones in the Tone-responsive channels (Figure 3C). HFA standard tone responses showed an earlier $50 \%$ area latency when compared to deviant responses in the insula (138 vs. $162 \mathrm{~ms}$ ) or STS (137 vs. $199 \mathrm{~ms}$ ), but not in STG (111 vs. $139 \mathrm{~ms}$ ). Figure 3D-F shows the mean fractional area latency for standard and deviant tones in the Condition-specific channels in insula, STS and STG cortices. Statistical details can be found in Section S.5.2 and Tables S2 and S3.

Eleven channels across 5 patients in STS, and 4 channels across 3 patients in STG presented HFA deviance detection effects. STG channels were sensitive to Intensity and Frequency dimensions only, whereas STS channels were sensitive to all dimensions (see details in section S.5.2, and condition-specific responses in STS and STG in Figure S5).

\section{DISCUSSION}

We examined the role of the human insular cortex in auditory processing focusing on deviance detection and HFA. The HFA results revealed auditory processing in the insular cortex, and automatic deviance detection in the inferior circular sulcus of the insula which was supported by additional ERP analysis.

\subsection{The insula as part of the auditory network}

Our results provide electrophysiological evidence that the insular cortex is involved in auditory processing with HFA indicating that the iCS and LG areas of the insula are most frequently activated. Previous studies have shown that posterior insula auditory HFA responses resemble those 
observed in Heschl's gyrus (Zhang et al., 2019), and that auditory perception is altered by electrical stimulation (Afif et al., 2010; Zhang et al., 2018) or focal insular strokes (Bamiou et al., 2003; Habib et al., 1995). Single unit responses in primate insular cortex have been shown to encode amplitude- and frequency modulated sounds (Bieser, 1998), and vocal communication sounds in its posterior part (Remedios et al., 2009). Additionally, the posterior insula is strongly connected to the primary- and association auditory areas, and the STS (Augustine et al., 1996; Ghaziri et al., 2017; Uddin, Nomi, Hébert-Seropian, Ghaziri, \& Boucher, 2017; Zhang et al., 2018), and its cytoarchitectonics is similar to that of cortical sensory areas (Rivier \& Clarke, 1997). Our findings are in line with prior evidence of auditory processing in the human and non-human primate insula.

\subsection{The role of the insula in automatic auditory deviance detection}

Prior studies suggested that the posterior part of the superior temporal plane is a key area for automatic auditory deviance detection by using iEEG (El Karoui et al., 2015; Halgren et al., 1995, Edwards et al., 2005; Kropotov et al., 1995; Rosburg et al., 2005), fMRI (Opitz, Rinne, Mecklinger, von Cramon, \& Schröger, 2002), and MEG/EEG source localization (Lappe et al., 2013; Rinne, Alho, Ilmoniemi, Virtanen, \& Näätänen, 2000). Other studies have implicated the frontal cortex in deviance detection (Bekinschtein et al., 2009; Liasis, Towell, Alho, \& Boyd, 2001; Phillips et al., 2016; Rosburg et al., 2005; Durschmid et al., 2106a, 2016b, Deouell. 2007). No iEEG study has reported data from the human insula during deviance detection.

Our HFA results indicate that the iCS is involved in automatic auditory deviance detection. The HFA responses of the iCS were sensitive to deviations in the frequency, intensity, location, and time dimensions. In agreement with our findings, both fMRI and lesion studies have suggested the involvement of the insular cortex in deviance detection. Griffiths et al. (1996) reported that a right insular stroke patient was unable to detect the auditory source of sound movements, and Bamiou et al. (2006) reported that unilateral insular stroke patients had deficits in temporal resolution and sequencing of sounds. fMRI studies reported that the left iCS responds to non-attended sound deviants in the frequency domain (Sabri et al., 2004), the left anterior insula responds to tone duration deviants (Schall et al., 2003), and there is a greater activation in the left insula for unexpected versus expected attended sounds (Nazimek et al., 2013). fMRI and lesion studies cannot rule out the possibility that the temporal cortices adjacent to the insula are contributing to the activations (fMRI) or functional deficits after lesion. In contrast, intracranial HFA obtained from a bipolar montage provides the spatial and temporal resolution necessary to localize the source and timing of local neural activity (Lachaux et al., 2012; Ray \& Maunsell, 2011; Rich \& Wallis, 2107; Watson et al., 2018). Our HFA analysis identified the iCS as the sub-insular area involved in deviance detection, showing its sensitivity to auditory deviance in multiple physical dimensions, and also delineated the temporal response profile. The longer latencies to deviants support unfolding processing when sensory predictions are violated.

At a group level, the Tone-Responsive channels showed differences between standard and deviant tones, and only a subgroup of these, the Condition-specific channels, were sensitive to specific deviant dimensions. We cannot rule out that the condition-specific effects observed in these channels were due to a better SNR.

Stimulus-specific adaptation (or habituation) may contribute to the results obtained for Int-Up, Freq-Up or Freq-Down deviants (Trautner et al., 2006). However, higher HFA responses to IntDown deviants in particular, but also to Loc-Left, Loc-Right, Time-Dur, and Time-Gap deviants 
cannot be explained as stimulus-specific adaptation. Since stimulus-specific adaptation and predictions are not mutually exclusive (Parras et al., 2017), and the deviant stimuli are unpredictable, we interpret the deviance detection effects as prediction error signals, which might include habituation effects in the Int-Up, Freq-Up or Freq-Down cases.

\subsection{Insula, Superior Temporal Sulcus and Superior Temporal Gyrus roles in auditory and deviance detection processing}

We observed auditory responses and deviance detection in STS and STG, and responses were later in insula when compared with STS and STG, suggesting that insula is engaged in hierarchical processing of auditory information. Notably, we observed later responses to deviants when compared to standards in the insula and STS cortices, but not in the STG.

\subsection{HFA responses and Event-related potentials}

The ERP findings support the HFA results. The strongest ERP responses were observed in the iCS, in accord with the HFA responses. The ERP Tone-responsive and Condition-specific channels were more widespread, observed in more patients, and mirrored the HFA findings (see for example Crone et al., 2001; Edwards et al., 2005; Durschmid et al., 2016). ERPs likely reflect the summation of synchronous long-duration and long-distance pre- and post-synaptic currents, in contrast to the short-duration and more local synaptic currents related to action potentials that underlie the HFA (Lachaux et al., 2012; Ray \& Maunsell, 2011; Manning et al., 2009; Watson et al., 2018). In our analysis, we used a bipolar montage to minimize the effect of volume conduction. However, the mitigation of far-field potentials is not total, and we cannot rule out the possibility that activity in the superior temporal plane contributed to the observed ERPs (Kajikawa \& Schroeder, 2011). We speculate that the different ERP responses observed in Group 1 and 2 are due to the combined effects of individual variability, different generators active in different time windows, and distances and orientations between the generators and the recording electrode pairs.

We observed early ERP components ( $\sim 70$ and $100 \mathrm{~ms})$, but statistical condition differences were only present later (> 100 ms; Celesia, 1971; Liégeois-Chauvel et al., 1994). MMN responses (Figure S3) were similar to the ones previously observed by depth intracranial recordings in the proximity of the auditory cortex (Kropotov et al., 1995; Halgren et al., 1995). Differences between the HFA response to standard and deviant tones were coincident with the intracranial equivalent and the typical scalp MMN's peak latency (Garrido et al., 2009; Näätänen et al., 2007), further supporting our ERP and HFA analysis on the role of the insula in auditory processing and deviance detection.

\section{DATA AVAILABILITY}

The conditions of our ethical approval do not permit public archiving of anonymized study data. Readers seeking access to the data supporting the claims in this paper should contact the corresponding author Alejandro Blenkmann; data manager Torgrim Mikal Langleite (t.m.langleite@psykologi.uio.no), Department of Psychology, University of Oslo; the Research Ethics Committee of El Cruce Hospital, Argentina; and the Regional Committees for Medical and Health Research Ethics, Region North Norway. Requests must meet the following specific conditions to obtain the data: a collaboration agreement, data sharing agreement, and a formal ethical approval. 
Experimental scripts and stimuli, and custom analysis code is available at https://osf.io/4u36q/

\section{ACKNOWLEDGMENTS}

We appreciate the patients for kindly participating in our study. We thank Sabine Leske, Julia Kam, and the members of the Knight Lab for valuable discussions. We want to express our gratitude to the EEG technicians at El Cruce Hospital and Oslo University Hospital-Rikshospitalet for their support. This work was partly supported by the Research Council of Norway (project number 240389), NINDS Grant R37NS21135, and NIMH CONTE Center P50MH109429.

\section{REFERENCES}

Afif, A., Minotti, L., Kahane, P., \& Hoffmann, D. (2010). Anatomofunctional organization of the insular cortex: A study using intracerebral electrical stimulation in epileptic patients. Epilepsia, 51(11), 2305-2315. https://doi.org/10.1111/j.1528-1167.2010.02755.x

Augustine, J. R. (1996). Circuitry and functional aspects of the insular lobe in primates including humans. Brain Research Reviews, 22(3), 229-244. https://doi.org/10.1016/S0165-0173(96)00011-2Ashburner, J., \& Friston, K. J. (2005). Unified segmentation. NeuroImage, 26(3), 839-851. https://doi.org/10.1016/j.neuroimage.2005.02.018

Bamiou, D. E., Musiek, F. E., \& Luxon, L. M. (2003). The insula (Island of Reil) and its role in auditory processing. Literature review. Brain Research. Brain Research Reviews, 42(2), 143-54. https://doi.org/10.1016/S01650173(03)00172-3

Bamiou, D. E., Musiek, F. E., Stow, I., Stevens, J., Cipolotti, L., Brown, M. M., \& Luxon, L. M. (2006). Auditory temporal processing deficits in patients with insular stroke. Neurology, 67(4), 614-619. https://doi.org/10.1212/01.wnl.0000230197.40410.db

Bekinschtein, T., Dehaene, S., Rohaut, B., Tadel, F., Cohen, L., \& Naccache, L. (2009). Neural signature of the conscious processing of auditory regularities. Proceedings of the National Academy of Sciences of the United States of America, 106(5), 1672-7. https://doi.org/10.1073/pnas.0809667106

Bieser, A. (1998). Processing of twitter-call fundamental frequencies in insula and auditory cortex of squirrel monkeys. Experimental Brain Research, 122(2), 139-148. https://doi.org/10.1007/s002210050501

Blenkmann, A. O., Phillips, H. N., Princich, J. P., Rowe, J. B., Bekinschtein, T. A., Muravchik, C. H., \& Kochen, S. (2017). iElectrodes: a comprehensive open-source toolbox for depth and subdural grid electrode localization. Frontiers in Neuroinformatics, 11(March), 14. https://doi.org/10.3389/FNINF.2017.00014

Bubic, A., von Cramon, D. Y., \& Schubotz, R .I. (2010). Prediction, cognition and the brain. Frontiers in Human Neuroscience, 4(March), 1-15. https://doi.org/10.3389/fnhum.2010.00025

Celesia, G. G. (1976). Organization of auditory cortical areas in man. Brain, 99(3), 403-414. https://doi.org/10.1093/brain/99.3.403

Clark, A. (2013). Whatever next? Predictive brains, situated agents, and the future of cognitive science. Behavioral and Brain Sciences (2013) 36, 181-253, 181-253. https://doi.org/10.1017/S0140525X12000477

Crone, N. E., Boatman, D., Gordon, B., \& Hao, L. (2001). Induced electrocorticographic gamma activity during auditory perception. Clinical Neurophysiology, 112(4), 565-582. https://doi.org/10.1016/S1388-2457(00)00545-9

Dale, A. M., Fischl, B., \& Sereno, M. I. (1999). Cortical surface-based analysis. I. Segmentation and surface reconstruction. NeuroImage, 9(2), 179-94. https://doi.org/10.1006/nimg.1998.0395

Deouell, L. Y. (2007). The frontal generator of the mismatch negativity revisited. Journal of Psychophysiology, 21(3-4), 188-203. https://doi.org/10.1027/0269-8803.21.34.188Destrieux, C., Fischl, B., Dale, A., \& Halgren, E. (2010). 
Automatic parcellation of human cortical gyri and sulci using standard anatomical nomenclature. NeuroImage, 53(1), 1-15. https://doi.org/10.1016/j.neuroimage.2010.06.010

Duvernoy, H. M. (1999). The Human Brain. Vienna: Springer Vienna. https://doi.org/10.1007/978-3-7091-6792-2

Dürschmid S, Edwards E, Reichert C, Dewar C, Hinrichs H, Heinze HJ, Kirsch HE, Dalal SS, Deouell LY, Knight RT. (2016a). Hierarchy of prediction errors for auditory events in human temporal and frontal cortex. Proc Natl Acad Sci U S A. 113(24), 6755-60. https://doi.org/10.1073/pnas.1525030113.

Dürschmid S, Zaehle T, Hinrichs H, Heinze HJ, Voges J, Garrido MI, Dolan RJ, Knight RT. (2016b). Sensory Deviancy Detection Measured Directly Within the Human Nucleus Accumbens. Cereb Cortex. 26(3):1168-1175. https://doi.org/10.1093/cercor/bhu304

Edwards, E., Soltani, M., Deouell, L. Y., Berger, M. S., \& Knight, R. T. (2005). High gamma activity in response to deviant auditory stimuli recorded directly from human cortex. Journal of Neurophysiology, 94(6), 4269-4280. https://doi.org/10.1152/jn.00324.2005

El Karoui, I., King, J.-R., Sitt, J., Meyniel, F., Van Gaal, S., Hasboun, D., ... Naccache, L. (2015). Event-Related Potential, Time-frequency, and Functional Connectivity Facets of Local and Global Auditory Novelty Processing: An Intracranial Study in Humans. Cerebral Cortex, 25(11), 4203-4212. https://doi.org/10.1093/cercor/bhu143

Fullerton, B. C., \& Pandya, D. N. (2007). Architectonic analysis of the auditory-related areas of the superior temporal region in human brain. The Journal of Comparative Neurology, 504(5), 470-498. https://doi.org/10.1002/cne.21432

Galaburda, A., \& Sanides, F. (1980). Cytoarchitectonic organization of the human auditory cortex. J-Comp-Neurol, 190(3), 597-610.

Garrido, M. I., Kilner, J. M., Stephan, K. E., \& Friston, K. J. (2009). The mismatch negativity: A review of underlying mechanisms. Clinical Neurophysiology, 120(3), 453-463. https://doi.org/10.1016/j.clinph.2008.11.029

Ghaziri, J., Tucholka, A., Girard, G., Houde, J. C., Boucher, O., Gilbert, G., ... Nguyen, D. K. (2017). The Corticocortical Structural Connectivity of the Human Insula. Cerebral Cortex (New York, N.Y. : 1991), 27(2), 1216-1228. https://doi.org/10.1093/cercor/bhv308

Griffiths, T. D., Rees, A., Witton, C., Shakir, R. A., Henning, G. B., \& Green, G. G. R. (1996). Evidence for a sound movement area in the human cerebral cortex. Nature, 383(6599), 425-427. https://doi.org/10.1038/383425a0

Habib, M., Daquin, G., Milandre, L., Royere, M. L., Rey, M., Lanteri, A., ... Khalil, R. (1995). Mutism and auditory agnosia due to bilateral insular damage-Role of the insula in human communication. Neuropsychologia, 33(3), 327-339. https://doi.org/10.1016/0028-3932(94)00108-2

Halgren, E., Baudena, P., Clarke, J. M., Heit, G., Liégeois, C., Chauvel, P., \& Musolino, a. (1995). Intracerebral potentials to rare target and distractor auditory and visual stimuli. I. Superior temporal plane and parietal lobe. Electroencephalography and Clinical Neurophysiology, 94(3), 191-220. Retrieved from http://www.ncbi.nlm.nih.gov/pubmed/7536154

Kajikawa, Y., \& Schroeder, C. E. (2011). How local is the local field potential? Neuron, 72(5), 847-858. https://doi.org/10.1016/j.neuron.2011.09.029

Kleiner M, Brainard D, P. D. (2007). What's new in Psychtoolbox-3? Perception, 36.

Kropotov, J. D., Nätnen, R., Sevostianov, A. V, Alho, K., Reinikainen, K., \& Kropotova, O. V. (1995). Mismatch negativity to auditory stimulus change recorded directly from the human temporal cortex. Psychophysiology, 32(4), 418-22. https://doi.org/10.1111/j.1469-8986.1995.tb01226.x

Lachaux, J. P., Axmacher, N., Mormann, F., Halgren, E., \& Crone, N. E. (2012). High-frequency neural activity and human cognition: Past, present and possible future of intracranial EEG research. Progress in Neurobiology, 98(3), 279-301. https://doi.org/10.1016/j.pneurobio.2012.06.008 
Lappe, C., Steinsträter, O., \& Pantev, C. (2013). Rhythmic and melodic deviations in musical sequences recruit different cortical areas for mismatch detection. Frontiers in Human Neuroscience, 7(June), 1-9.

https://doi.org/10.3389/fnhum.2013.00260

Liasis, A., Towell, A., Alho, K., \& Boyd, S. (2001). Intracranial identification of an electric frontal-cortex response to auditory stimulus change: A case study. Cognitive Brain Research, 11(2), 227-233. https://doi.org/10.1016/S09266410(00)00077-X

Menon, V., \& Uddin, L. Q. (2010). Saliency, switching, attention and control: a network model of insula function. Brain Structure \& Function, 214(5-6), 655-667. https://doi.org/10.1007/s00429-010-0262-0

Müller, B. W., Jüptner, M., Jentzen, W., \& Müller, S. P. (2002). Cortical activation to auditory mismatch elicited by frequency deviant and complex novel sounds: A PET study. NeuroImage, 17(1), 231-239.

https://doi.org/10.1006/nimg.2002.1176

Näätänen, R., Paavilainen, P., Rinne, T., \& Alho, K. (2007). The mismatch negativity (MMN) in basic research of central auditory processing: a review. Clinical Neurophysiology : Official Journal of the International Federation of Clinical Neurophysiology, 118(12), 2544-90. https://doi.org/10.1016/j.clinph.2007.04.026

Näätänen, R., Pakarinen, S., Rinne, T., \& Takegata, R. (2004). The mismatch negativity (MMN): Towards the optimal paradigm. Clinical Neurophysiology, 115, 140-144. https://doi.org/10.1016/j.clinph.2003.04.001

Nazimek, J. M., Hunter, M. D., Hoskin, R., Wilkinson, I., \& Woodruff, P. W. (2013). Neural basis of auditory expectation within temporal cortex. Neuropsychologia, 51(11), 2245-2250.

https://doi.org/10.1016/j.neuropsychologia.2013.07.019

Oostenveld, R., Fries, P., Maris, E., \& Schoffelen, J.-M. (2011). FieldTrip: Open Source Software for Advanced Analysis of MEG, EEG, and Invasive Electrophysiological Data. Computational Intelligence and Neuroscience, 2011, 1-9. https://doi.org/10.1155/2011/156869

Opitz, B., Rinne, T., Mecklinger, A., von Cramon, D. Y., \& Schröger, E. (2002). Differential Contribution of Frontal and Temporal Cortices to Auditory Change Detection: fMRI and ERP Results. NeuroImage, 15(1), 167-174. https://doi.org/10.1006/nimg.2001.0970

Parras, G. G., Nieto-Diego, J., Carbajal, G. V., Valdés-Baizabal, C., Escera, C., \& Malmierca, M. S. (2017). Neurons along the auditory pathway exhibit a hierarchical organization of prediction error. Nature Communications, $8(1)$. https://doi.org/10.1038/s41467-017-02038-6

Phillips, H. N., Blenkmann, A., Hughes, L. E., Bekinschtein, T. a., \& Rowe, J. B. (2015). Hierarchical Organization of Frontotemporal Networks for the Prediction of Stimuli across Multiple Dimensions. Journal of Neuroscience, 35(25), 9255-9264. https://doi.org/10.1523/JNEUROSCI.5095-14.2015

Phillips, H. N., Blenkmann, A., Hughes, L. E., Kochen, S., Bekinschtein, T. A., Cam-CAN, \& Rowe, J. B. (2016). Convergent evidence for hierarchical prediction networks from human electrocorticography and magnetoencephalography. Cortex, 82, 192-205. https://doi.org/10.1016/j.cortex.2016.05.001

Phillips, H. N., Blenkmann, A., Hughes, L. E., Kochen, S., Bekinschtein, T. A., Cam, C. A. N., \& Rowe, J. B. (2016). Convergent evidence for hierarchical prediction networks from human electrocorticography and magnetoencephalography. Cortex, 82, 192-205. https://doi.org/10.1016/j.cortex.2016.05.001

Ray, S., \& Maunsell, J. H. R. (2011). Different Origins of Gamma Rhythm and High-Gamma Activity in Macaque Visual Cortex, 9(4). https://doi.org/10.1371/journal.pbio.1000610

Remedios, R., Logothetis, N. K., \& Kayser, C. (2009). An Auditory Region in the Primate Insular Cortex Responding Preferentially to Vocal Communication Sounds. Journal of Neuroscience, 29(4), 1034-1045. https://doi.org/10.1523/JNEUROSCI.4089-08.2009

Rich, E. L., \& Wallis, J. D. (2017). Spatiotemporal dynamics of information encoding revealed in orbitofrontal highgamma. Nature Communications, 8(1), 1-13. https://doi.org/10.1038/s41467-017-01253-5 
Rinne, T., Alho, K., Ilmoniemi, R. J., Virtanen, J., \& Näätänen, R. (2000). Separate Time Behaviors of the Temporal and Frontal Mismatch Negativity Sources. NeuroImage, 12(1), 14-19. https://doi.org/10.1006/nimg.2000.0591

Rivier, F., \& Clarke, S. (1997). Cytochrome oxidase, acetylcholinesterase, and NADPH-diaphorase staining in human supratemporal and insular cortex: Evidence for multiple auditory areas. NeuroImage, 6(4), 288-304. https://doi.org/10.1006/nimg.1997.0304

Rosburg, T., Trautner, P., Dietl, T., Korzyukov, O. a, Boutros, N. N., Schaller, C., ... Kurthen, M. (2005). Subdural recordings of the mismatch negativity (MMN) in patients with focal epilepsy. Brain : A Journal of Neurology, 128(Pt 4), 819-28. https://doi.org/10.1093/brain/awh442

Sabri, M., Kareken, D. A., Dzemidzic, M., Lowe, M. J., \& Melara, R. D. (2004). Neural correlates of auditory sensory memory and automatic change detection. NeuroImage, 21(1), 69-74. https://doi.org/10.1016/j.neuroimage.2003.08.033

Schall, U., Johnston, P., Todd, J., Ward, P. B., \& Michie, P. T. (2003). Functional neuroanatomy of auditory mismatch processing: An event-related fMRI study of duration-deviant oddballs. NeuroImage, 20(2), 729-736. https://doi.org/10.1016/S1053-8119(03)00398-7

Studholme, C., Hill, D. L. G., \& Hawkes, D. J. (1999). An overlap invariant entropy measure of 3D medical image alignment. Pattern Recognition, 32(1), 71-86. https://doi.org/10.1016/S0031-3203(98)00091-0

Trautner, P., Rosburg, T., Dietl, T., Fell, J., Korzyukov, O. A., Kurthen, M., ... Boutros, N. N. (2006). Sensory gating of auditory evoked and induced gamma band activity in intracranial recordings. NeuroImage, 32(2), 790-798. https://doi.org/10.1016/j.neuroimage.2006.04.203

Uddin, L. Q., Nomi, J. S., Hébert-Seropian, B., Ghaziri, J., \& Boucher, O. (2017). Structure and Function of the Human Insula. Journal of Clinical Neurophysiology: Official Publication of the American Electroencephalographic Society, 34(4), 300-306. https://doi.org/10.1097/WNP.0000000000000377

Watson, B. O., Ding, M., \& Buzsáki, G. (2018). Temporal coupling of field potentials and action potentials in the neocortex. European Journal of Neuroscience, (October 2017), 1-16. https://doi.org/10.1111/ejn.13807

Zhang, Y., Zhou, W., Wang, S., Zhou, Q., Wang, H., Zhang, B., ... Wang, X. (2019). The Roles of Subdivisions of Human Insula in Emotion Perception and Auditory Processing. Cerebral Cortex, (June), 1-12. https://doi.org/10.1093/cercor/bhx334 


\section{FIGURES \& TABLES}

Table 1

\begin{tabular}{|c|c|c|c|c|c|}
\hline $\begin{array}{l}\text { Mismatch } \\
\text { Dimension }\end{array}$ & Condition & Frequencies & Intensity & $\begin{array}{l}\text { Perceived } \\
\text { sound source } \\
\text { location }\end{array}$ & Time \\
\hline & Standard & $500,1000,1500 \mathrm{~Hz}$ & $A_{S}$ & Midline & Duration $75 \mathrm{~ms}$ \\
\hline Frequency & $\begin{array}{l}\text { Freq-High } \\
\text { Freq-Low }\end{array}$ & $\begin{array}{c}550,1100,1650 \mathrm{~Hz} \\
450,900,1350 \mathrm{~Hz}\end{array}$ & & & \\
\hline Intensity & $\begin{array}{l}\text { Int-Up } \\
\text { Int-Down }\end{array}$ & & $\begin{array}{l}+10 \mathrm{~dB} \\
-10 \mathrm{~dB}\end{array}$ & & \\
\hline Location & $\begin{array}{l}\text { Loc-Left } \\
\text { Loc-Right }\end{array}$ & & & $\begin{array}{l}\text { Left } \\
\text { Right }\end{array}$ & \\
\hline Time & $\begin{array}{l}\text { Time-Dur } \\
\text { Time-Gap }\end{array}$ & & & & $\begin{array}{c}\text { Duration } 25 \mathrm{~ms} \\
7 \mathrm{~ms} \text { gap in the middle }\end{array}$ \\
\hline
\end{tabular}

Stimulus features. Empty cells indicate same features as standard tones.

Tones were presented at a comfortable level to participants. The intensity of deviant tones $\left(A_{D}\right)$ is expressed in decibels $(\mathrm{dB})$ relative to the standard tone intensity $\left(\mathrm{A}_{\mathrm{S}}\right)$, i.e. Intensity Deviant $=10$ $\log _{10}\left(A_{D} / A_{S}\right)$.

Perceived sound location was manipulated by introducing a time lag of $\pm 800 \mu$ s between the left and right ear.
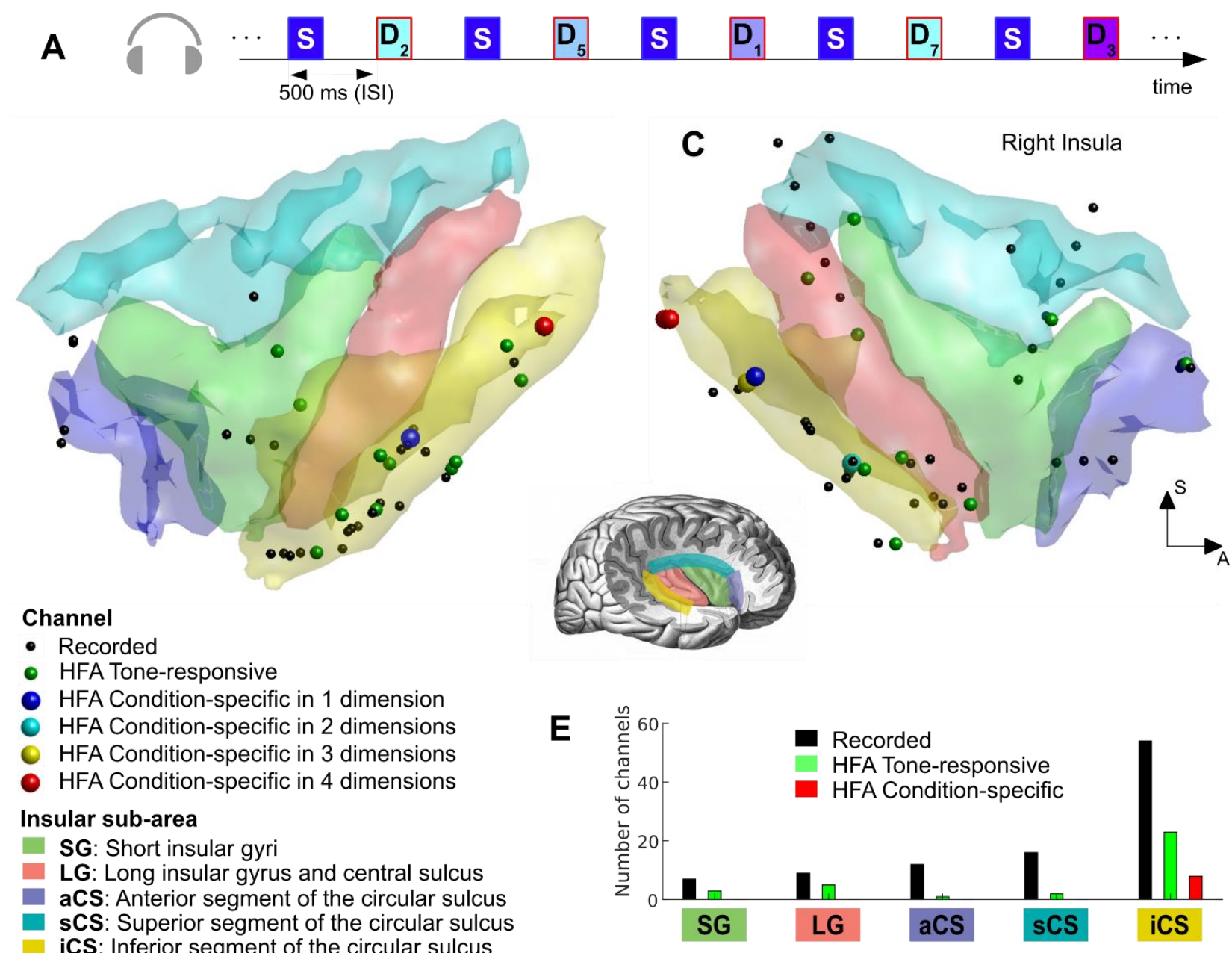

Figure 1. Experimental design and spatial distribution of recorded, HFA Tone-responsive, and HFA Condition-specific channels. 
A. Experimental design denoting standard and deviant tones are interleaved. Deviants in different physical dimensions (frequency, intensity, location and time) were randomly distributed. See deviant probabilities and physical characteristics in Table 1. ISI denotes inter-stimulus interval.

B \& C. Channels from all subjects are shown over a 3D reconstruction of the left and right insular cortex. Recorded channels are represented by small black spheres, tone responsive channels by medium size green spheres, and condition-specific channels by big blue, cyan, yellow, and red spheres. The color-coding for Condition-specific channels indicate the number of physical dimensions (deviant features) to which the channel showed statistical effects according to legend "Channels". The anatomical reconstruction with subdivisions was obtained from a segmentation of the MRI brain scan of one patient (Patient 13). Insular subdivisions are color-coded according to legend "Insular sub-area". Each sphere is located in the mean coordinate of a pair of electrodes constituting a bipolar channel.

D. Illustrative map of the insular subdivisions (modified from Textbook and Atlas of Human Anatomy; Sobotta, 1908). Insular subdivisions are color-coded according to legend "Insular subarea".

E. Distribution of recorded, responsive, and Condition-specific channels by anatomical area.
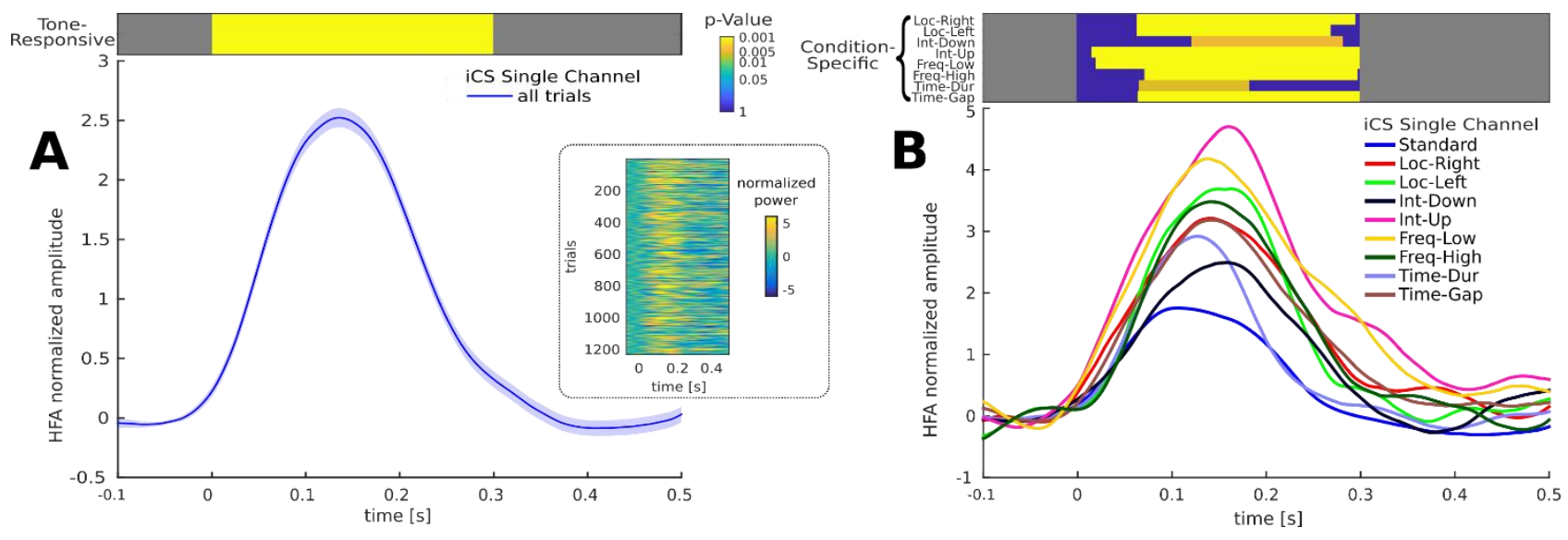

Figure 2. HFA responses in the insula.

A. Mean HFA response to all tones in one illustrative Tone-responsive channel in the iCS (see Methods, section Tone-responsive channels for definition). Inset shows the single trials HFA time courses. The top image shows the period of HFA with significantly higher amplitude than at baseline ( $p$-values obtained from permutation test).

B. Mean HFA response to each condition in one Condition-specific channel in the iCS. The top image denotes the periods where HFA responses to deviants were significantly higher than the standard tone responses ( $p$-values obtained from post hoc permutation test).

Shadowed areas depict mean \pm SEM. Non-tested periods are depicted in gray. 
A

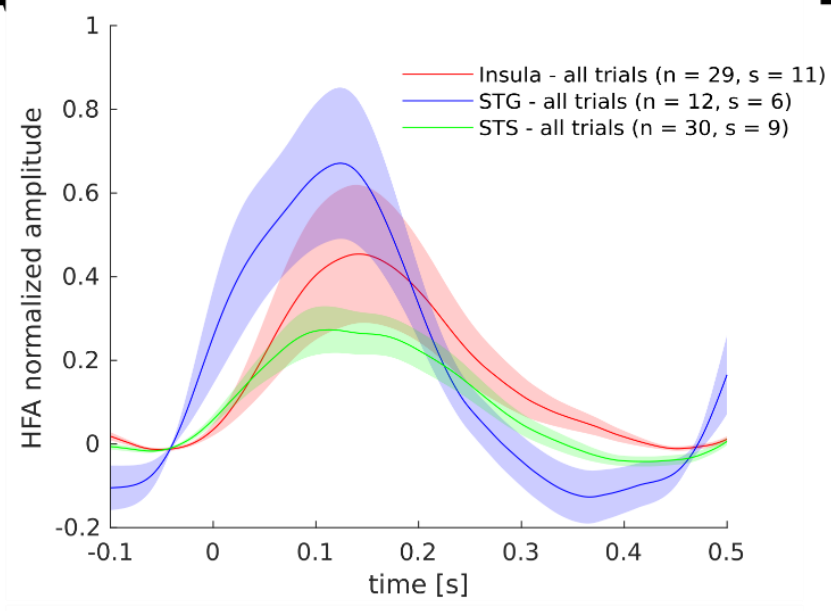

C

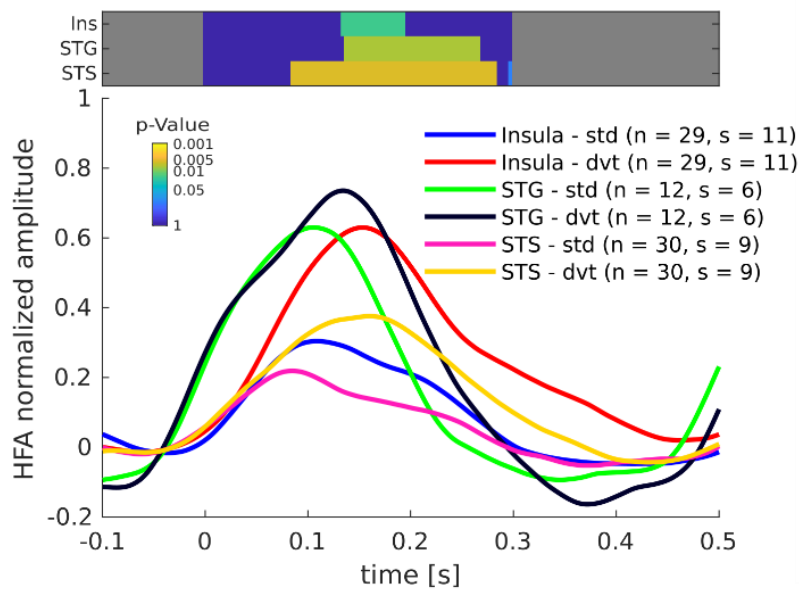

$\mathbf{E}$

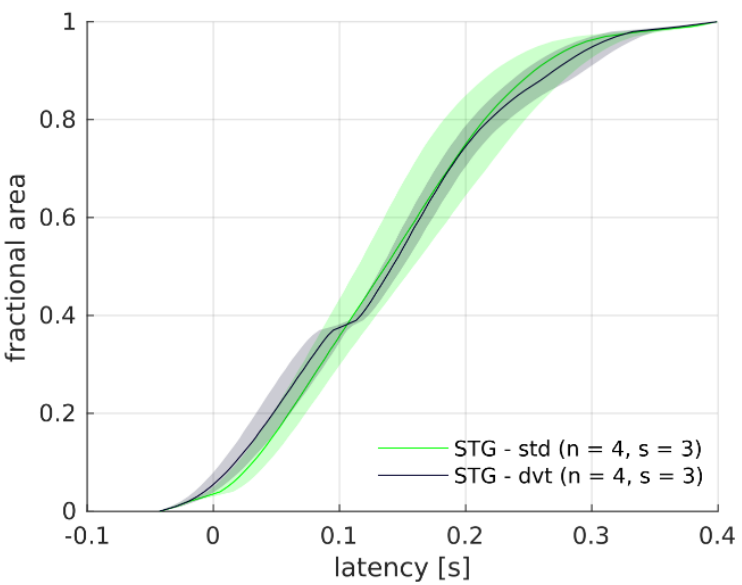

B

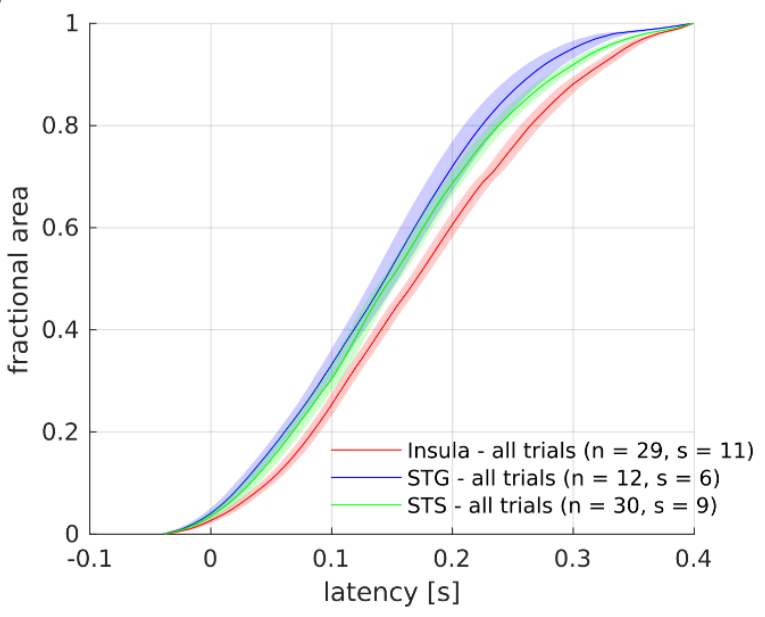

D

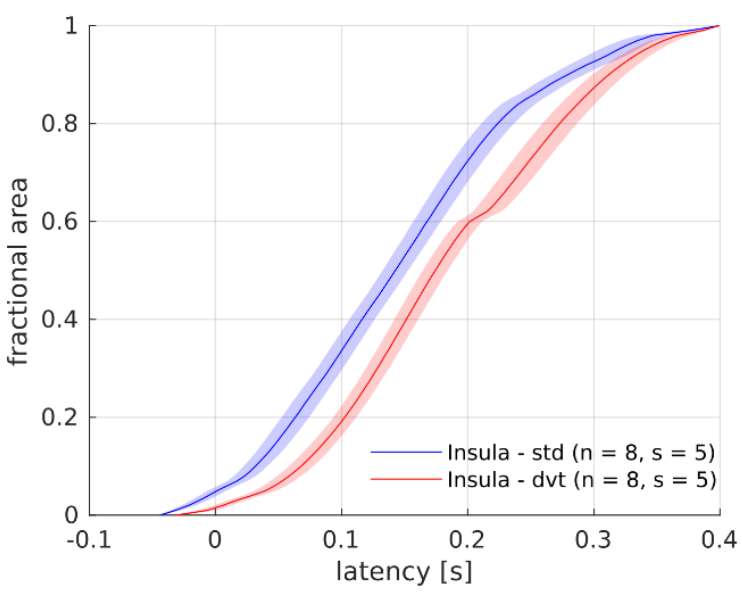

$\mathbf{F}$

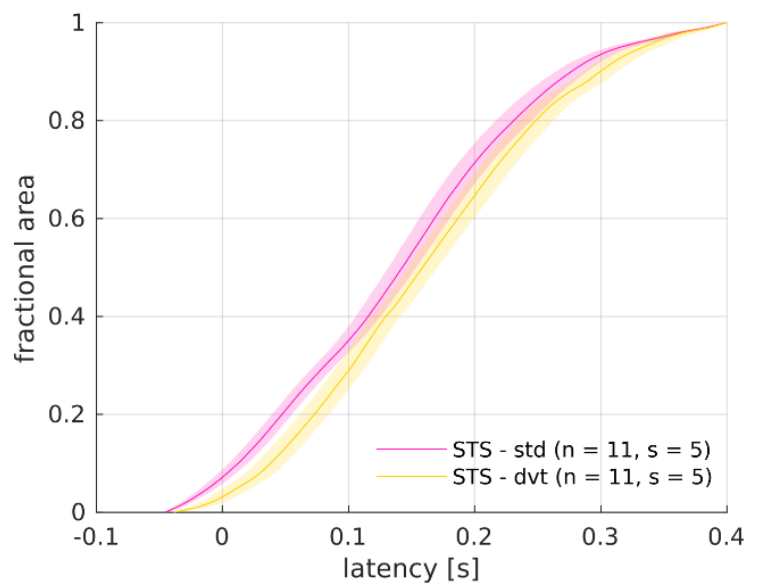

Figure 3. HFA responses in the Insula, Superior Temporal Sulcus, and Superior Temporal Gyrus.

A. Mean HFA auditory responses in the Tone-responsive channels across subjects in the insular, STS, and STG cortices. B. Mean fractional area latency for responses to tones in the Tone-responsive channels in the Insula, STG and STS cortices. The 50\% fractional area latency in the insular cortex was later than in STS and STG cortices. See Table S2 for statistical testing details. C. Mean HFA responses to standard and deviant tones in the Tone-Responsive channels across subjects in the insular, STS, and STG cortices. Image on top shows statistical differences between standard and deviant tones in each area (134-196 ms period $\mathrm{p}=0.014$ for insula, $137-269 \mathrm{~ms}$ period $\mathrm{p}=0.007$ for STG, and $85-285 \mathrm{~ms}$ period $\mathrm{p}=0.005$ for STS, cluster-based permutation t-test). Non-tested periods are depicted in gray. D. Mean fractional area latency for responses to standard and deviant tones in the insular cortex Condition-specific channels. The 50\% fractional area latency to standard tones was 
earlier than for deviant tones. See Table S3 for statistical testing details. E. Mean fractional area latency for responses to standard and deviant tones in the STS Condition-specific channels. The 50\% fractional area latency to standard tones was earlier than for deviant tones. See Table S3 for statistical testing details. F. Mean fractional area latency for responses to standard and deviant tones in the STG Condition-specific channels. No statistical differences were observed in the $50 \%$ fractional area latency responses to standard and deviant tones.

Shadowed areas depict mean \pm SEM. $n$ indicates the number of channels averaged, $s$ indicates the number of subjects, Std indicates Standard, and Dvt indicates Deviant.
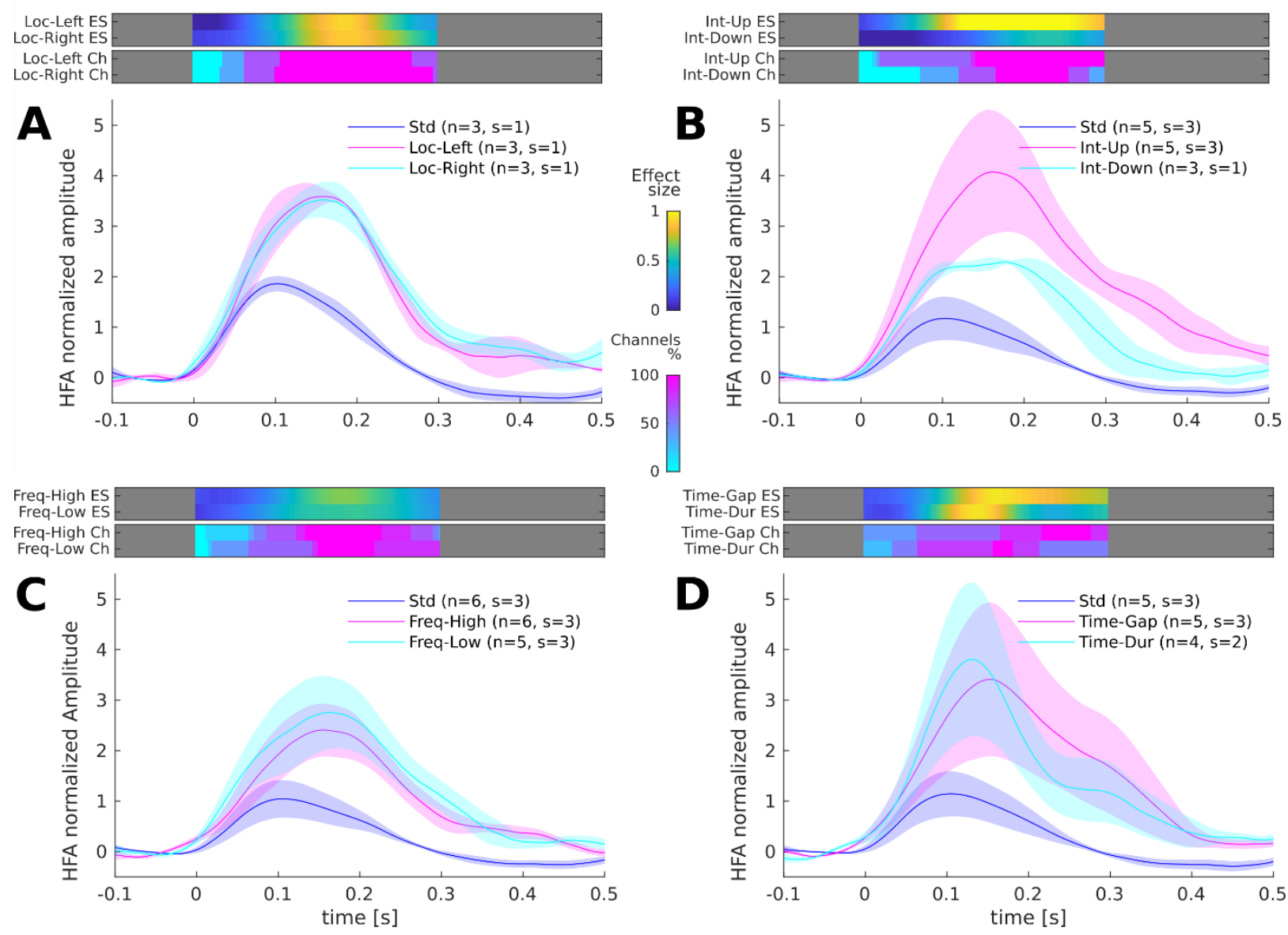

Figure 4. HFA Condition-specific responses in the inferior segment of the circular sulcus of the insula.

Responses where averaged across channels from different subjects showing significant differences between the deviant and standard conditions.

A. Mean HFA responses to standard (blue) and location deviant tones (Loc-Left, magenta and LocRight, cyan).

B. Mean HFA responses to standard (blue) and intensity deviant tones (Int-Up, magenta and IntDown, cyan).

C. Mean HFA responses to standard (blue) and frequency deviant tones (Freq-High, magenta and Freq-Low, cyan).

D. Mean HFA responses to standard (blue) and time deviant tones (Time-Gap, magenta and TimeDur, cyan).

The top images in each subplot show the averaged Cohen's d effect size (ES) for each condition across channels (top row), and the number of channels showing statistically significant conditionspecific effects (bottom row).

Shadowed areas depict mean \pm SEM. $n$ indicates the number of channels averaged, $s$ indicates the number of subjects, and Std indicates Standard. Non-tested periods are depicted in gray. 
Channels group 1

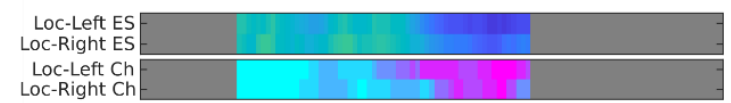

A
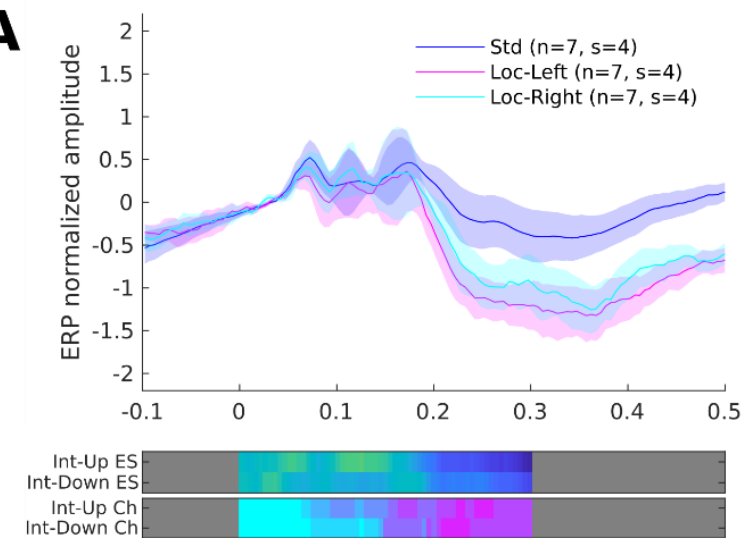

C

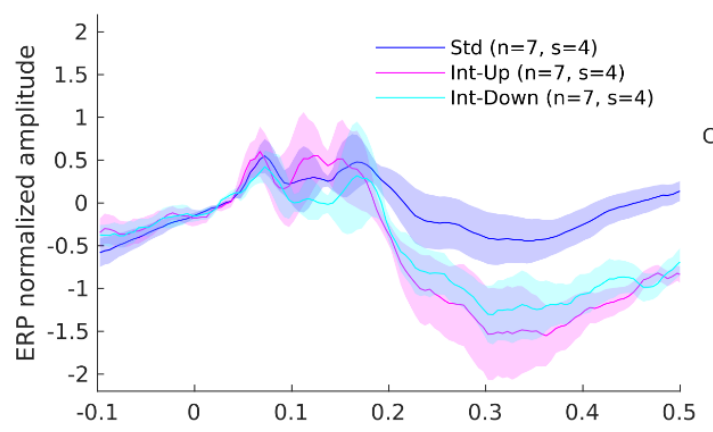

Freq-High ES
Freq-Low ES

Freq-High Ch

E
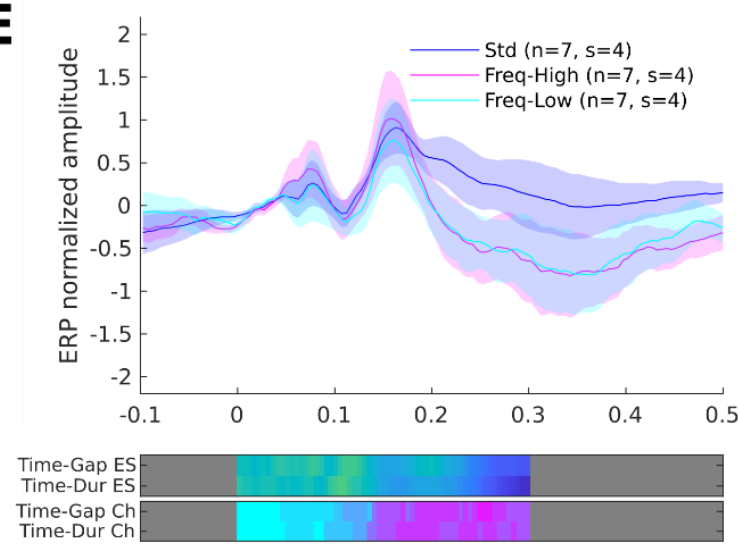

$\mathbf{G}$

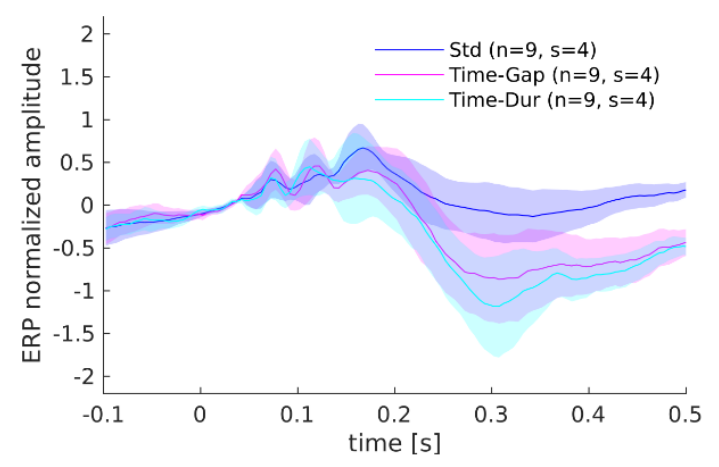

Channels group 2

Loc-Left ES
Loc-Right ES

Loc-Left Ch
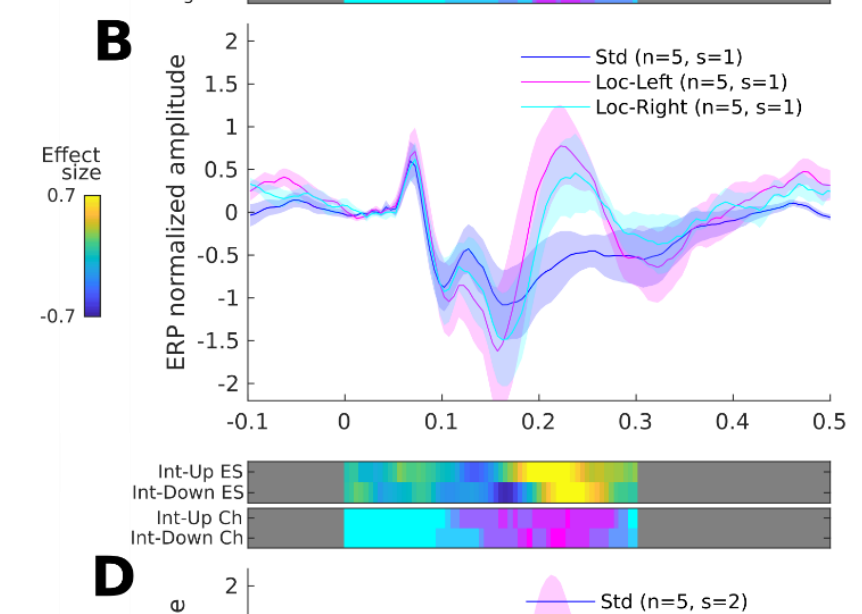

D

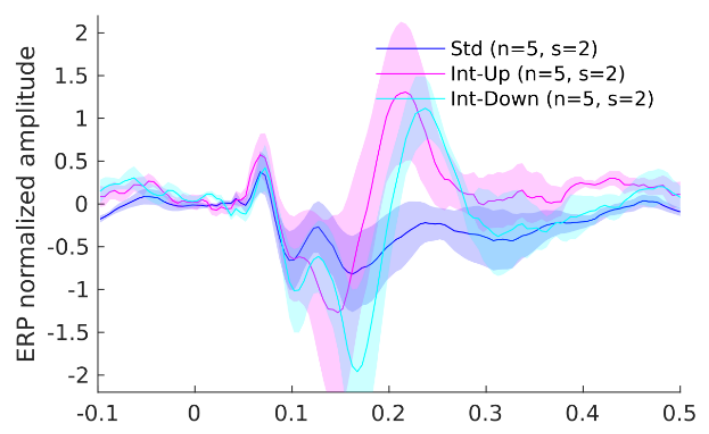

Freq-High ES

Freq-High Ch

F
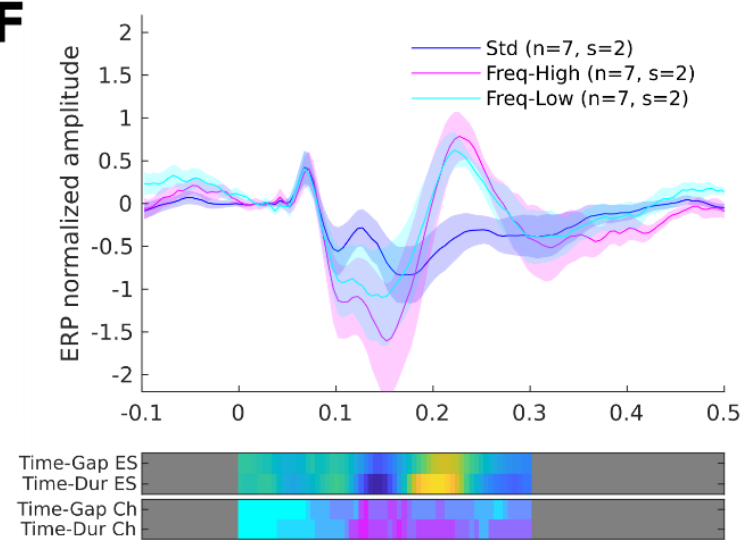

H

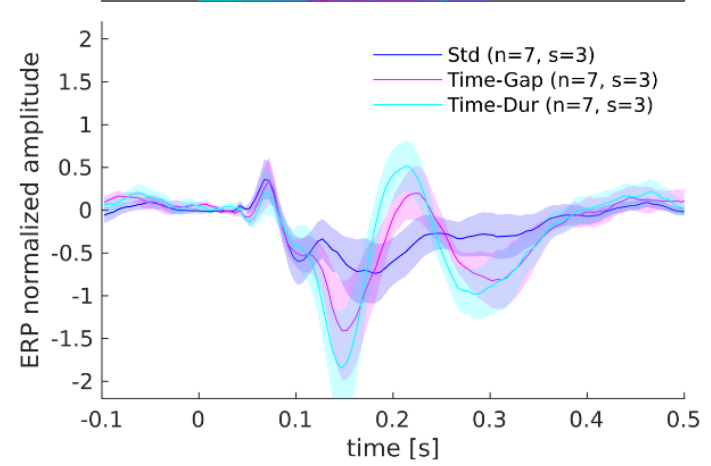


Figure 5. ERP Condition-specific responses in the inferior segment of the circular sulcus of the insula.

ERP responses where averaged across channels from different subjects showing significant differences between the deviant and standard conditions. For visualization purposes, responses were grouped and polarity aligned according to PCA weights (see section S.3.3 for details). Only channels that showed significant responses to both conditions within each dimension of analysis were included in the plots in order to obtain comparable waveforms.

Responses for Group 1 are on the left column (A, C, E, and G) and responses for Group 2 are on the right column ( $\mathrm{B}, \mathrm{D}, \mathrm{F}$, and $\mathrm{H})$.

A \& B. Mean ERP responses to standard (blue) and location deviant tones (Loc-Left, magenta and Loc-Right, cyan).

C \& D. Mean ERP responses to standard (blue) and intensity deviant tones (Int-Up, magenta and IntDown, cyan).

E \& F. Mean ERP responses to standard (blue) and frequency deviant tones (Freq-High, magenta and Freq-Low, cyan). G \& H. Mean ERP responses to standard (blue) and time deviant tones (Time-Gap, magenta and Time-Dur, cyan).

The top images in each subplot show the averaged Cohen's d effect size (ES) for each condition across channels (top row), and the percentage of channels showing statistically significant condition-specific effects (bottom row).

Shadowed areas depict mean \pm SEM. $\mathrm{n}$ indicates the number of channels averaged, and $\mathrm{s}$ indicates the number of subjects. Non-tested periods are depicted in gray. 


\section{Supplementary material}

\section{$\underline{\text { S.1 Demographic characteristics of patients and number of recording channels }}$}

\begin{tabular}{|c|c|c|c|c|c|c|c|c|c|}
\hline $\begin{array}{l}\text { Pati } \\
\text { ent }\end{array}$ & $\operatorname{Sex}$ & Age & $\begin{array}{l}\text { Ins } \\
\text { S } \\
\text { G }\end{array}$ & $\begin{array}{l}\text { sula } \\
\text { L } \\
\text { G }\end{array}$ & $\begin{array}{l}\mathbf{r} \text { ch } \\
\mathbf{a} \\
\mathrm{C} \\
\mathrm{S}\end{array}$ & $\begin{array}{l}\mathrm{ann} \\
\mathrm{sC} \\
\mathrm{S}\end{array}$ & $\begin{array}{l}\text { els* } \\
\text { iC } \\
\text { S }\end{array}$ & $\begin{array}{c}\text { Total } \\
\text { insular } \\
\text { channels }\end{array}$ & $\begin{array}{l}\text { Temporal } \\
\text { channels }\end{array}$ \\
\hline 1 & $\mathrm{M}$ & 20 & & & & & 2 & 2 & 4 \\
\hline 2 & $\mathrm{~F}$ & 22 & 1 & 3 & & 1 & 4 & 6 & 3 \\
\hline 3 & $\mathrm{M}$ & 19 & & & & & 3 & 3 & 2 \\
\hline 4 & $\mathrm{~F}$ & 49 & & 2 & & & 6 & 8 & 7 \\
\hline 5 & $M$ & 19 & & & 2 & & 3 & 5 & \\
\hline 6 & $\mathrm{M}$ & 21 & 3 & & & & 5 & 8 & 10 \\
\hline 7 & $\mathrm{M}$ & 25 & & & & & 2 & 2 & 7 \\
\hline 8 & $\mathrm{M}$ & 37 & & & & & 5 & 5 & 10 \\
\hline 9 & $\mathrm{~F}$ & 23 & & & 3 & & & 3 & 11 \\
\hline 10 & $\mathrm{M}$ & 38 & 3 & 1 & & 2 & & 3 & 1 \\
\hline 11 & $\mathrm{~F}$ & 30 & & & 2 & & 8 & 10 & 5 \\
\hline 12 & $\mathrm{M}$ & 27 & & & & & 3 & 3 & 14 \\
\hline 13 & $\mathrm{~F}$ & 34 & & & & & 6 & 6 & 4 \\
\hline 14 & $\mathrm{M}$ & 38 & & 3 & 5 & 11 & 4 & 21 & 9 \\
\hline 15 & $\mathrm{M}$ & 48 & & & & 1 & & 1 & \\
\hline 16 & $\mathrm{~F}$ & 50 & & & & 1 & 3 & 4 & 25 \\
\hline $\begin{array}{l}\text { Tota } \\
1\end{array}$ & $\begin{array}{l}6 \mathrm{~F} / 10 \\
\mathrm{M}\end{array}$ & 31.3 & 7 & 9 & 12 & 16 & 54 & 90 & 112 \\
\hline
\end{tabular}

Table S1. Demographic characteristics of implanted patients and number of channels in regions of interest within the insular and temporal cortex. Bipolar channels contained signals from two insular cortex electrodes or one insular and one white matter electrode. (*)Notice that 8 channels were recorded from two insular sub-area electrodes and therefore the Total number of channels (last column) might not be equal to the sum of the channels from the individual sub-areas (columns 4 to 8 ). SG: short insular gyri, LG: long insular gyrus and central sulcus of the insula, aCS: anterior segment of the circular sulcus of the insula, sCS: superior segment of the circular sulcus of the insula, iCS: inferior segment of the circular sulcus of the insula.

\section{S.2 Insular HFA analysis. Additional results}

\section{S.2.1 Deviant effects: The HFA condition-specific channels}

For comparisons with other studies, we report the mean coordinates $(\mathrm{x}, \mathrm{y}, \mathrm{z})$ in MNI space of the left and right Condition-specific insular channels to be located at $(-42,-15,5)$ and $(42,-16,-3)$.

\section{$\underline{\text { S.3 ERP Methods }}$}

\section{S.3.1 Low Frequency activity time series extraction}

Preprocessed data were bandpass filtered from 0.01 to $30 \mathrm{~Hz}$. The mean amplitude of the baseline period ( -100 to $0 \mathrm{~ms}$ ) of each trial was removed from the entire trial, thereby computing the Low Frequency (LF) time series of each channel relative to the baseline activity. The polarity of the signals that constitute a bipolar channel affects the polarity of the LF time series.

In order to later compare responses from different subjects, all trial time series were divided by the standard deviation pulled from all trials in the baseline period, thereby computing the low frequency 
time series of each channel as a normalized measure that was relative to the variability of the baseline activity (-100 to $0 \mathrm{~ms}$ relative to onset).

\section{S.3.2 Tone-responsive ERP channels}

For the LF time series, the same procedure as for HFA was applied to define ERP tone-responsive channels, but testing both positive and negative ERP deflections (two-tailed test). Channels were considered responsive if the median ERP response to tones, irrespective of type, was statistically higher or lower in the post-stimulus period (0 to $300 \mathrm{~ms}$ after stimulus onset) relative to the baseline period (-100 to $0 \mathrm{~ms}$ ) (Durschmid et al., 2016a). We tested the differences in five $100 \mathrm{~ms}$ overlapping windows in the post-stimulus period, with $50 \%$ overlap, using a permutation-based approach. For each channel, we computed the difference between the ERP medians in the post-stimulus windows and the ERP medians in the baseline period. We then created an empirical distribution by circularshifting of the ERP trial time series (between -100 and $500 \mathrm{~ms}$ ) by a random number of samples. This allows any time-locked neural activity to be teased apart. Differences between the medians in the post-stimulus and baseline periods were measured for each surrogate set of trials. The procedure was repeated 1000 times and a null distribution of differences was built. Channels exceeding the 97.5th percentile, or below the 2.5 th percentile of the channel-specific surrogate distribution in any of the five windows were considered showing a significant time-locked ERP modulation and were marked as Tone-responsive channels.

Due to the bipolar channel recordings, we expected to see polarity inversions in the ERP signals. To be able to visualize the most relevant peaks across channels, independent of their polarity, we computed the instantaneous power of the normalized responses.

\section{S.3.3 Differences in ERP responses to Standard and Deviant tones. Clustering channel responses by PCA weighting}

ERP responses to standard and deviant tones in the tone-responsive channels were analyzed.

First, we observed that responses in the $400-500 \mathrm{~ms}$ period after tone onset, that were used as a baseline for the next trial, were different for standard and deviant tones, building a systematic difference between conditions in the time period that was used as baseline for the following trial (100 to $0 \mathrm{~ms}$ ). To remove this systematic "leakage" from the previous trial in the baseline period of the trial under analysis, we redefined the baseline period to 0-50 ms after the tone onset (Hahne and Friederici, 2001, Lansbergenet al., 2007). This correction is based on the assumption that the corrected ERP time-courses are similar within the 0-50 ms time period after the tone onset.

Using principal component analysis (PCA) we identified groups of ERP responses with consistent temporal patterns across channels and subjects (in a similar approach to Durschmid et al., 2016). PCA also allowed us to address polarity reversals. First, we computed the ERP difference signal between the average response to deviants and the average response to standard tones for all the Toneresponsive channels. Then, we performed a PCA of the differential signal and considered the first components that explained $75 \%$ of the variance. Finally, we grouped the ERP responses from different channels according to the highest weights of the PCA decomposition. In other words, the channels that weighted more on the first component were grouped together, the channels that weighted more on the second component were grouped together, and so on for each component. In addition, to deal with polarity changes, we multiplied the signal of each channel by the sing present in the weighting (loading) matrix.

Please note that we analyzed the ERP signals, and not the components of the PCA analysis. In other words, the ERP responses were grouped according to their PCA loads, in some cases with a polarity change.

Two-tailed t-tests were performed to determine if the ERP responses to deviants were higher or lower in amplitude than those to standards within each group of channels (cluster-based permutation test, alpha $=0.05,1000$ permutations). 


\section{S.3.4 Condition-specific ERP channels}

For the LF time series, the same procedure as for HFA was applied, but testing both positive and negative ERP deflections (two-tailed test).

For each channel, we performed four one-way ANOVA cluster-based permutation tests with 3 levels: the standard tones and the two deviant tones of each dimension (alpha $=0.05,1000$ permutations), to determine whether the Tone-responsive channels were sensitive to intensity, frequency, location or time deviances. Post-hoc two-tailed t-tests were performed to determine if the ERP responses to deviants were higher or lower in amplitude than those to standards (cluster-based permutation test, alpha $=0.05,1000$ permutations).

Statistical testing was done on individual channels. However, in order to visualize results, we grouped channels using the same criteria as defined before (S.3.3). To summarize the Condition-specific effects from individual channel statistics, we computed the average of Cohen's d effect size across channels.

\section{$\underline{\text { S.4 ERP results }}$}

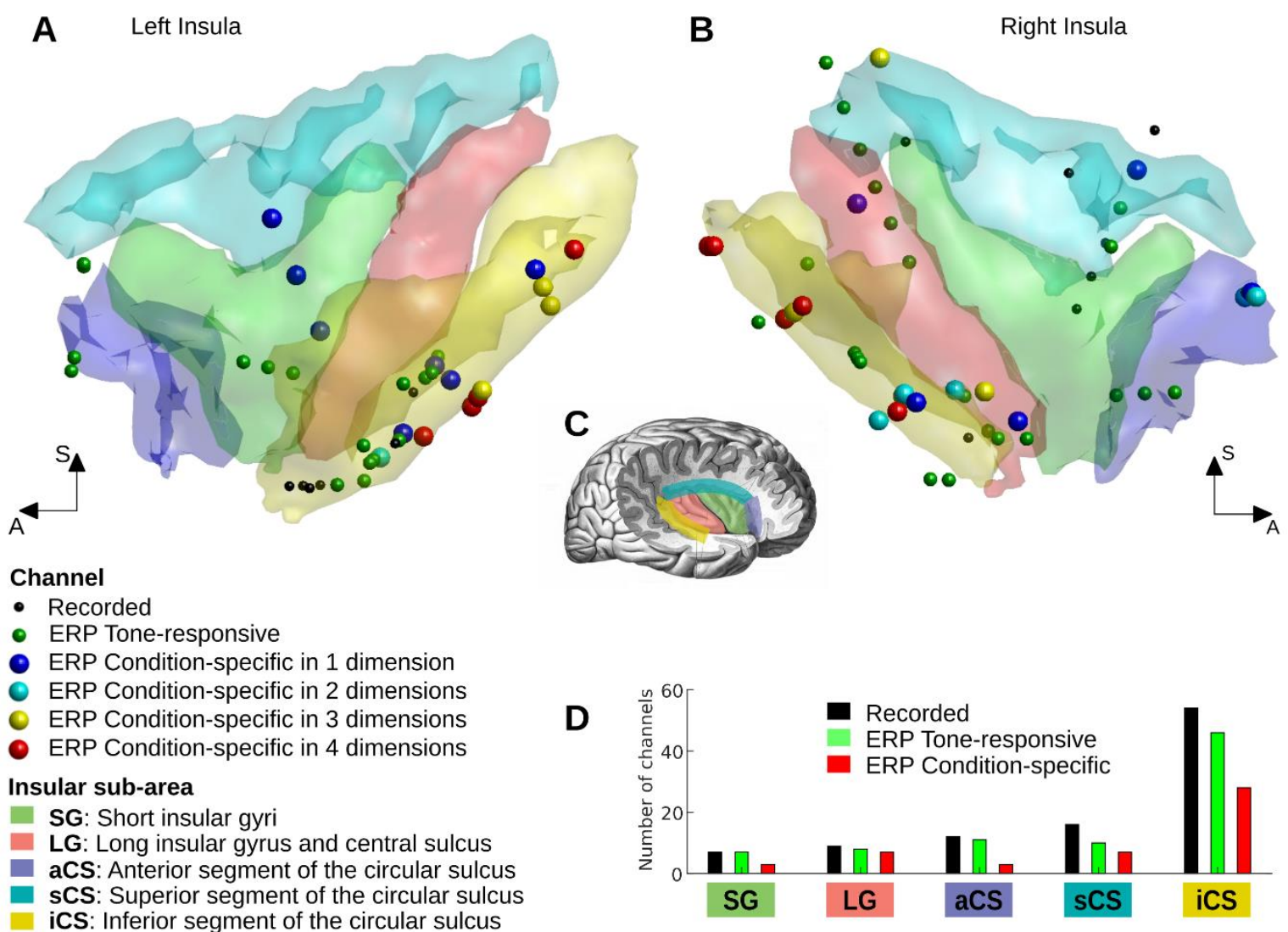

Figure S1. Spatial distribution of recorded, responsive and task-condition specific channels for the ERP analysis.

A \& B. Channels from all subjects are shown over a 3D reconstruction of the left and right insular cortex. Recorded channels are represented by small black spheres, tone responsive channels by medium size green spheres, and task-condition specific channels by big blue, cyan, yellow, and red spheres. The color-coding for Condition-specific channels indicates the number of physical dimensions to which the channel showed statistical effects according to the legend "Channels". The anatomical reconstruction with subdivisions was obtained from a segmentation of the MRI brain scan of one patient (Patient 13). Insular subdivisions are color-coded according to legend "Insular sub-area". C. Illustrative map of the insular subdivisions, modified from Textbook and Atlas of Human Anatomy (Sobotta, 1908). Insular subdivisions are color-coded according to the legend "Insular sub-area". D. Distribution of recorded, responsive, and Condition-specific channels by anatomical area. 


\section{S.4.2 Auditory effects: The ERP Tone-responsive channels}

The ERP analysis indicated auditory effects in 76 channels across 15 patients (permutation test, $\mathrm{p}<$ $0.05)$. Figure $\mathrm{S} 1$ shows the anatomical distribution of these tone-responsive channels. To describe the general dynamics of these responses, we first computed the mean normalized power response (Figure S2A). Since we observed that the individual ERP responses across different areas were not consistent, we computed the mean normalized power by insular sub-area (Figure S2B). The responses from the iCS were more predominant than the other areas. Given that the iCS is the area where we observed all the condition-specific responses in the HFA analysis and the strongest ERP responses, we focused the subsequent ERP analysis only on this area.
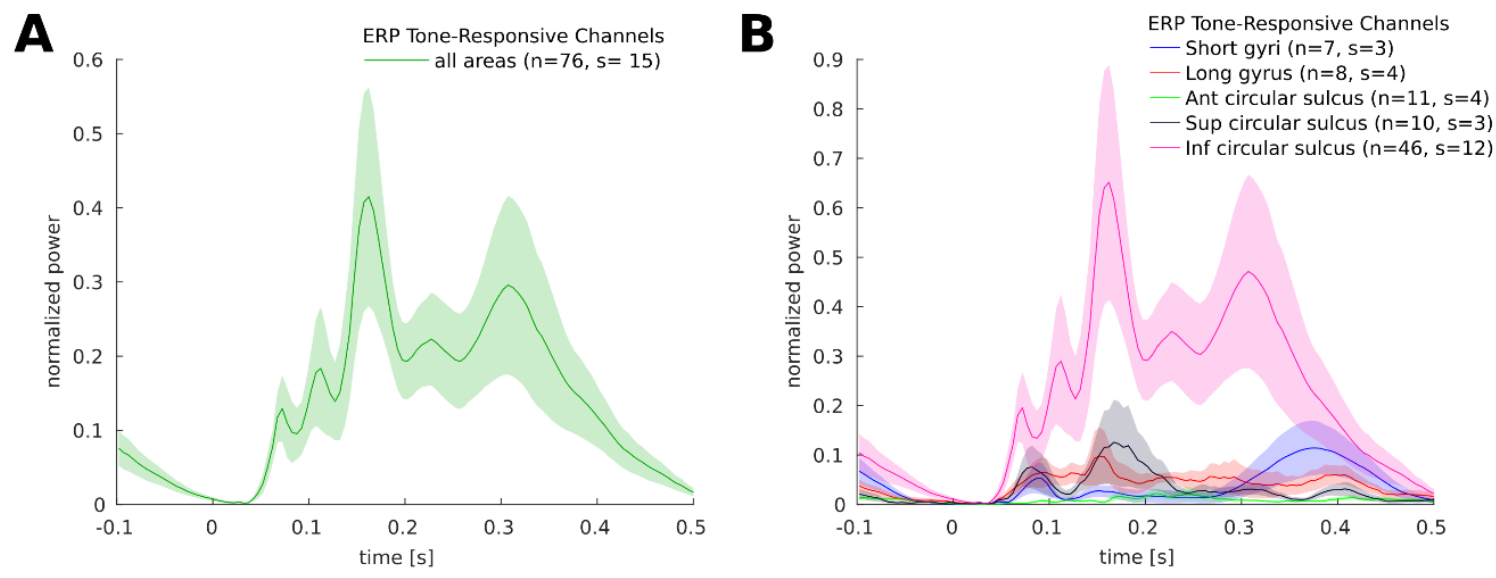

Figure S2 Power of the ERP response to tones

A. Mean normalized power of the ERP responses to tones across all responsive channels from different subjects in all studied areas. The presence of five peaks is observed at 72, 112, 162, 227, and $307 \mathrm{~ms}$.

B. Mean normalized power of the ERP responses to tones across all responsive channels from different subjects for each individual insula sub-area. Notice the higher amplitude of the inferior segment of the circular sulcus of the insula.

Shadowed areas depict mean \pm SEM. $n$ indicates the number of channels averaged, $s$ indicates the number of subjects.

\section{S.4.3 Differences in ERP responses to Standard and Deviant tones. Clustering iCS channel responses by PCA weighting}

Given the variability of the ERP responses across channels and subjects, and to overcome the changes of polarity observed, we performed a PCA analysis of the signal difference between responses to standard and deviant tones from all the iCS responsive channels.

The $70 \%$ of the explained variance threshold indicated that only the first two components of the PCA needed to be considered to group the responses. Therefore, we grouped the ERP responses into two groups, Group 1 and Group 2, according to the highest load of the PCA decomposition in each of these components, and multiplied the ERP signals by the sign of the corresponding component load.

Responses in Group 1 (Figure S3A) were slow, with a peak around $300 \mathrm{~ms}$. A difference between responses to standard and deviant conditions was observed $200-300 \mathrm{~ms}$ after tone onset.

Responses in Group 2 (Figure S3B) were in general faster, with multiple peaks. Peaks at 72 and 102 ms were consistent for both conditions. After this period, standard and deviant tone responses showed different peaks: 127 and 173 ms peaks for standards, and 152 and $222 \mathrm{~ms}$ for deviants. Statistical differences between conditions were observed $\sim 100-160 \mathrm{~ms}$, and $\sim 200-260 \mathrm{~ms}$ after onset. The average difference between standard and deviant tone responses, i.e. the intracranial equivalent of the scalp mismatch negativity (MMN) waveform, shows two clear peaks of opposite polarity at 142 and $217 \mathrm{~ms}$.

The previously described MMN potential observed in scalp EEG recordings for the same Optimum1 paradigm had two clear peaks at $\sim 150$ and 250 ms (Naatanen et al., 2004), which might correspond to the ones described here in Group 2 responses. 

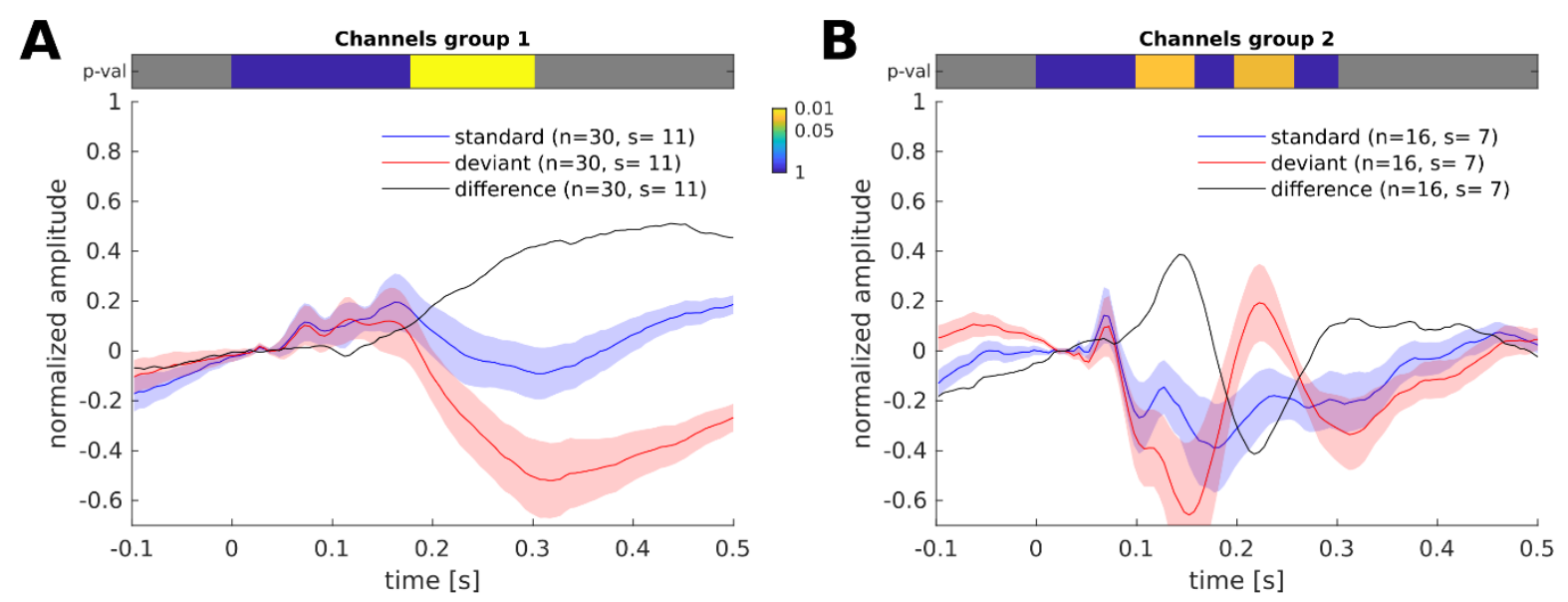

Figure S3. ERP responses to Standard and Deviant tones in the inferior segment of the circular sulcus of the insula. ERP responses to Standard and Deviant tones grouped according to PCA weights (see details in section S.3.3). Channels were signcorrected based on these loads. Images on top show statistical differences between Standard and Deviant tones. A. Group 1 channels show a later difference in the response to Standards and Deviants, in the $180-300 \mathrm{~ms}$ period $(\mathrm{p}=0.003$, cluster-based permutation $\mathrm{t}$ test). B. Group 2 channels show a difference in the response to Standards and Deviants in the 102-157 ms, and 202-257 ms time period $(\mathrm{p}=0.019$ and $\mathrm{p}=0.023$ respectively, cluster-based permutation $\mathrm{t}$-test $)$.

Shadowed areas depict mean \pm SEM. $n$ indicates the number of channels averaged, $s$ indicates the number of subjects.

\section{S.4.4 Deviance detection effects: The ERP Condition-specific channels}

ERP condition-specific effects were present in 48 channels, in 12 patients (permutation test, $\mathrm{p}<0.05$ ). Condition-specific channels were observed in the short gyrus $(n=3)$, long gyrus $(n=7)$, anterior segment of the circular sulcus $(n=2)$, superior segment of the circular sulcus $(n=7)$, and inferior segment of the circular sulcus $(n=28)$. Figure S1 shows the spatial distribution of these channels. We observed Condition-specific effects to Loc-Left in 21 channels, Loc-Right in 19 channels, FreqHigh in 15 channels, Freq-Low in 16 channels, Int-Up in 22 channels, Int-Down in 15 channels, TimeGap in 17 channels, and Time-Dur in 19 channels.

\section{S.5. HFA responses in the superior temporal cortex. A comparison to the insular cortex responses.}

\section{S.5.1 Region of interest channel selection}

We compared the auditory and deviance detection processing in the insular cortex with the temporal cortex.

We defined as ROI (see section 2.8) superior temporal sulcus (STS) and superior temporal gyrus (STG, composed by i) Lateral aspect of the superior temporal gyrus, ii) Planum polare of the superior temporal gyrus, and iii) Temporal plane of the superior temporal gyrus). We extracted 112 channels in the superior temporal cortex across 14 subjects (see Table S1 for details). We selected channels located only in STS $(n=93)$, or only in the STG $(n=19)$ for subsequent analysis.

\section{S.5.2 Latency analysis}

To analyze the latency of the HFA responses, we used the fractional area latency method (Luck, 2005). The area under the average HFA responses for each channel and condition were normalized between 0 and 1 , within the $-50 \mathrm{~ms}$ to $400 \mathrm{~ms}$ temporal window, given that some channels, mainly in STG, showed pre-stimulus onset activations.

We statistically tested the latency of the HFA responses at the $50 \%$ fractional area latency.

Table S2 shows 50\% area latency results for the responses in the Tone-responsive channels. 
Table S2

\begin{tabular}{|c|c|c|c|c|c|}
\hline $\begin{array}{l}\text { Condition } \backslash \\
\text { Brain area }\end{array}$ & & $\begin{array}{c}\text { Insula } \\
(\mathrm{n}=29, \mathrm{~s}=11)\end{array}$ & $\begin{array}{c}\text { STS } \\
(\mathrm{n}=30, \mathrm{~s}=9)\end{array}$ & $\begin{array}{c}\text { STG } \\
(\mathrm{n}=12, \mathrm{~s}=6)\end{array}$ & $\begin{array}{c}\text { Statistical testing } \\
\text { (Wilcoxon rank-sum test) }\end{array}$ \\
\hline \multirow{2}{*}{ All Tones } & median & $164 \mathrm{~ms}$ & $133 \mathrm{~ms}$ & $140 \mathrm{~ms}$ & \multirow{2}{*}{$\begin{array}{l}\text { Ins }>\text { STS }(p=0.016) \\
\text { Ins }>\text { STG }(p=0.021)\end{array}$} \\
\hline & $95 \% \mathrm{CI}$ & $143-192 \mathrm{~ms}$ & $117-167 \mathrm{~ms}$ & $118-190 \mathrm{~ms}$ & \\
\hline \multirow{2}{*}{ Standard Tones } & median & $151 \mathrm{~ms}$ & $127 \mathrm{~ms}$ & $122 \mathrm{~ms}$ & \multirow{2}{*}{$\begin{array}{l}\text { Ins }>\text { STS }(p=0.021) \\
\text { Ins }>\text { STG }(p=0.109)\end{array}$} \\
\hline & $95 \% \mathrm{CI}$ & $133-172 \mathrm{~ms}$ & $117-156 \mathrm{~ms}$ & $104-210 \mathrm{~ms}$ & \\
\hline \multirow{2}{*}{ Deviant Tones } & median & $169 \mathrm{~ms}$ & $141 \mathrm{~ms}$ & $142 \mathrm{~ms}$ & \multirow{2}{*}{$\begin{array}{l}\text { Ins }>\text { STS }(p=0.069) \\
\text { Ins }>\text { STG }(p=0.028)\end{array}$} \\
\hline & $95 \% \mathrm{CI}$ & $155-199 \mathrm{~ms}$ & $128-169 \mathrm{~ms}$ & $130-172 \mathrm{~ms}$ & \\
\hline
\end{tabular}

$50 \%$ area latency for HFA responses in insular cortex (Ins), superior temporal sulcus (STS), and superior temporal gyrus (STG) in Tone-responsive channels. CI denotes confidence interval, $n$ indicates the number of channels, and $s$ indicates the number of subjects.

Table S3 shows the 50\% area latency results for Condition-specific channels.

\section{Table S3}

\begin{tabular}{|l|l|c|c|c|}
\hline Condition \Brain area & \multicolumn{1}{|c|}{$\begin{array}{c}\text { Insula } \\
(\mathrm{n}=8, \mathrm{~s}=5)\end{array}$} & $\begin{array}{c}\text { STS } \\
(\mathrm{n}=11, \mathrm{~s}=5)\end{array}$ & $\begin{array}{c}\text { STG } \\
(\mathrm{n}=4, \mathrm{~s}=3)\end{array}$ \\
\hline Standard tones & median & $138 \mathrm{~ms}$ & $137 \mathrm{~ms}$ & $111 \mathrm{~ms}$ \\
\cline { 2 - 5 } & $95 \% \mathrm{CI}$ & $119-161 \mathrm{~ms}$ & $117-179 \mathrm{~ms}$ & $104-210 \mathrm{~ms}$ \\
\hline Deviant Tones & median & $162 \mathrm{~ms}$ & $199 \mathrm{~ms}$ & $139 \mathrm{~ms}$ \\
\cline { 2 - 5 } & $95 \% \mathrm{CI}$ & $151-205 \mathrm{~ms}$ & $132-218 \mathrm{~ms}$ & $121-165 \mathrm{~ms}$ \\
\hline $\begin{array}{l}\text { Statistical testing } \\
\text { (Wilcoxon signed rank test) }\end{array}$ & $\begin{array}{c}\text { Dvt }>\mathrm{Std} \\
(\mathrm{p}=0.019)\end{array}$ & $\begin{array}{c}\text { Dvt }>\mathrm{Std} \\
(\mathrm{p}<0.001)\end{array}$ & $\begin{array}{c}\text { Dvt }>\text { Std } \\
(\mathrm{p}=0.437)\end{array}$ \\
\hline
\end{tabular}

$50 \%$ area latency for HFA responses to Standard (Std) and Deviant (Dvt) tones in insular cortex, superior temporal sulcus (STS), and superior temporal gyrus (STG) in Condition-specific channels. CI denotes confidence interval, $n$ indicates the number of channels, and $s$ indicates the number of subjects.

Figures S4 shows the fractional area latency for standard and deviant tones in the insula, STS and STG cortices for the Tone-responsive channels.

A

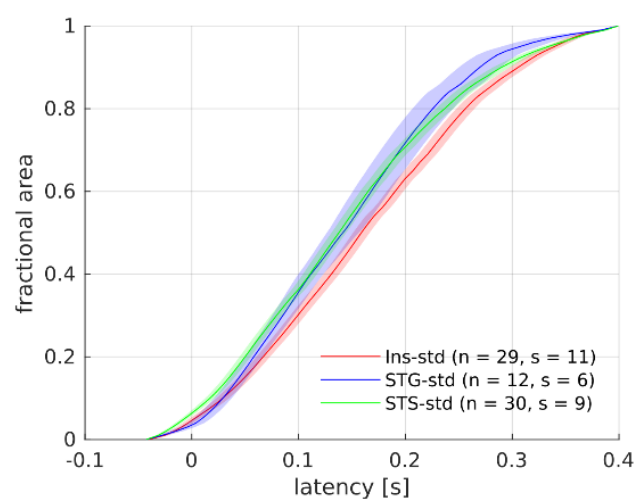

B

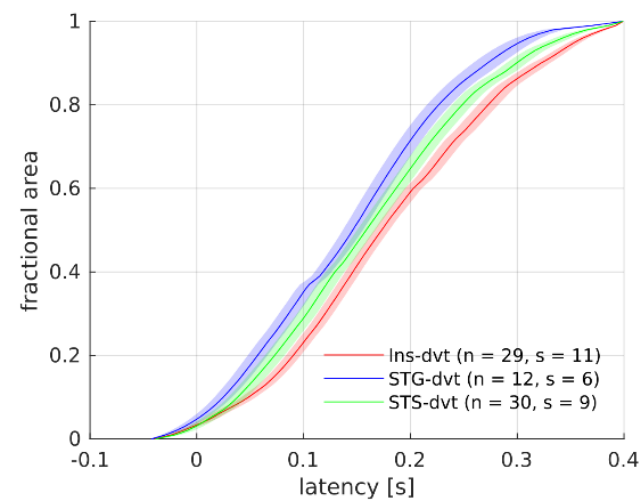

Figure S4 (previous page). Mean fractional area latency for HFA responses to standard and deviant tones.

A. Mean fractional area latency for responses to standard tones in Tone-responsive channels located in the Insula, STG and STS. B. Mean fractional area latency for responses to deviant tones in Tone-responsive channels located in the Insula, STG and STS.

Note that the latencies corresponding to 50\% fractional area are the ones tested in tables S2 and S3.

Shadowed areas depict mean \pm SEM. $n$ indicates the number of channels averaged, $s$ indicates the number of subjects. 


\section{S.5.3 Deviance detection effects: Comparison between Condition-specific HFA responses in Insula,}

STS, and STG

A total of 11 Condition-specific channels were observed in STS across 5 subjects, and 4 channels in STG across 3 subjects. Figure S5 shows the mean HFA condition-specific responses to Location, Intensity, Frequency, and Time dimensions for STS and STG channels obtained from different subjects. We observed no condition-specific responses to Location or Time in the STG. However, overall we observed similar proportions of Condition-specific channels relative to the Toneresponsive in the insula, STS and STG areas (28\%, 37\%, and 33\%, respectively).

To summarize the condition-specific effects from individual channel statistics, we computed the average of Cohen's d effect size across channels (Figure S5, top images). Effect sizes above medium (0.5) were observed for most conditions (Cohen, 1992). Compared to the insula HFA conditionspecific responses (Figure 4), the STS and STG responses showed smaller effect sizes.

Figure S5 (Next page). Condition-specific HFA responses in the STS and STG.

Responses were averaged across channels from different subjects showing significant differences between the deviant and standard conditions. The left column shows responses in STS, and the right column shows responses in STG.

The top images in each subplot show the averaged Cohen's d effect size for each condition across channels (top), and the percentage of channels showing statistically significant condition-specific effects (second row).

Shadowed areas depict mean \pm SEM. $n$ indicates the number of channels, $s$ indicates the number of subjects, and Std indicates Standard. 


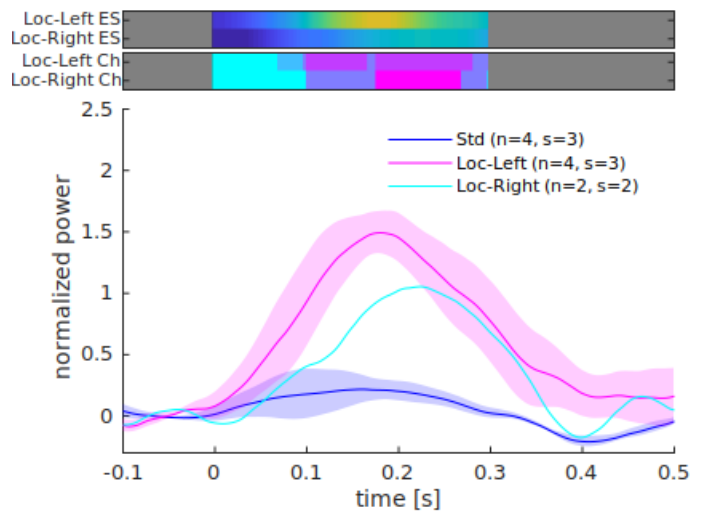

No CS responses in the Location dimension
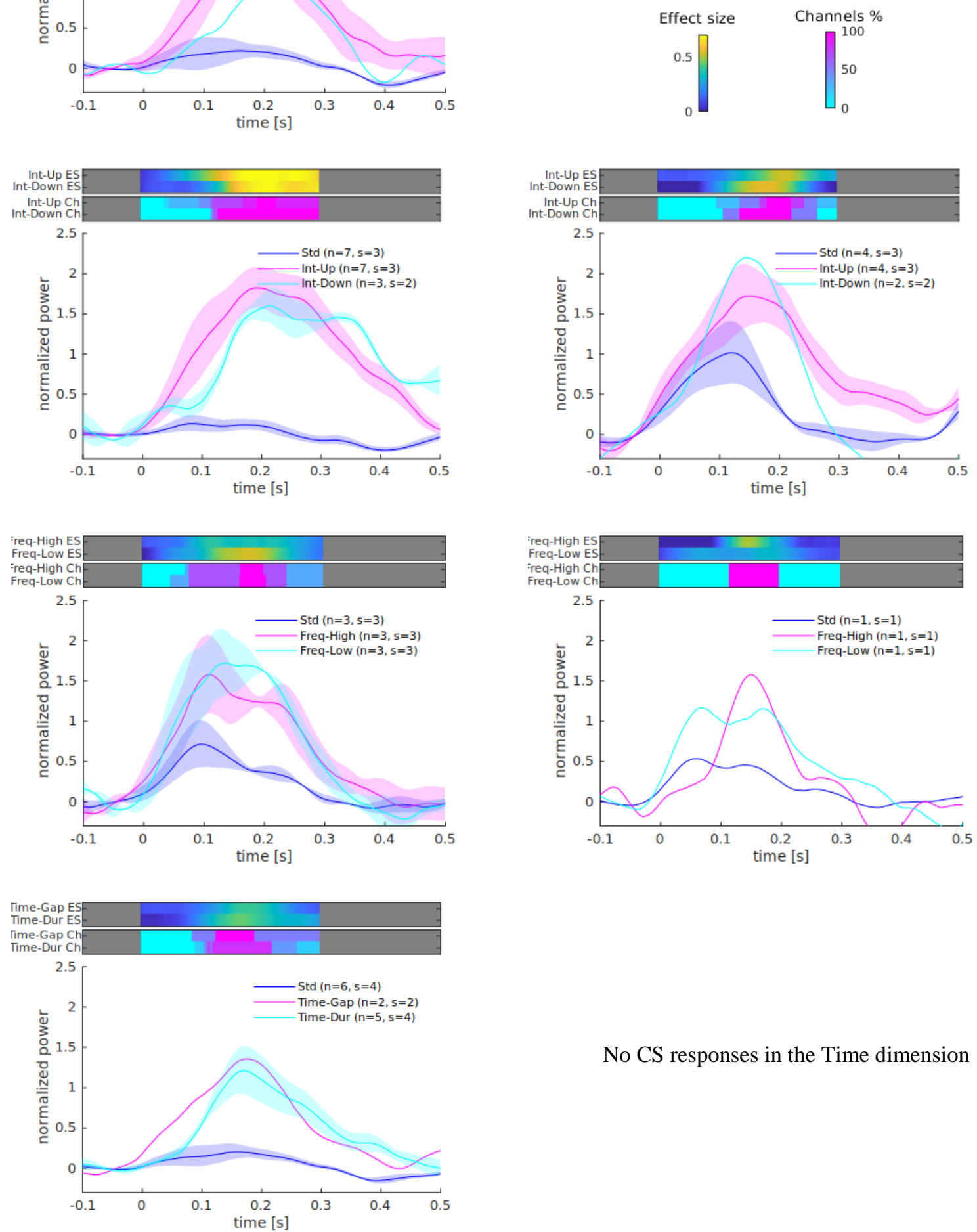

No CS responses in the Time dimension 


\section{References}

Cohen, J. (1992). A power primer. Psychological Bulletin, 112(1), 155-159.

https://doi.org/10.1037/0033-2909.112.1.155

Dürschmid, S., Edwards, E., Reichert, C., Dewar, C., Hinrichs, H., Heinze, H.-J... Knight, R. T. (2016). Hierarchy of prediction errors for auditory events in human temporal and frontal cortex. Proceedings of the National Academy of Sciences, 113(24), 201525030.

https://doi.org/10.1073/pnas.1525030113

Hahne, A., \& Friederici, A. D. (2001). Processing a second language: Late learners' comprehension mechanisms as revealed by event-related brain potentials. Bilingualism: Language and Cognition, 4(2), 123-141.

Lansbergen, M. M., Bocker, K. B., Bekker, E. M., \& Kenemans, J. L. (2007). Neural correlates of stopping and self-reported impulsivity. Clin Neurophysiol, 118(9), 2089-2103.

Luck, S. J. (2005). An Introduction to the Event-Related Potential Technique. MIT Press.

Näätänen, R., Pakarinen, S., Rinne, T., \& Takegata, R. (2004). The mismatch negativity (MMN):

Towards the optimal paradigm. Clinical Neurophysiology, 115, 140-144.

https://doi.org/10.1016/j.clinph.2003.04.001 